OPEN ACCESS

Edited by:

Gary James Harfitt,

The University of Hong Kong,

Hong Kong

Reviewed by:

Vânia Galindo Massabni,

University of São Paulo, Brazil

Joseph Samuel Backman,

Alpine School District, United States

*Correspondence:

Sylvie Lidolf

sylvie.lidolf@univ-fcomte.fr

Specialty section:

This article was submitted to

Teacher Education,

a section of the journal

Frontiers in Education

Received: 10 January 2020

Accepted: 19 March 2020

Published: 12 May 2020

Citation:

Lidolf S and Pasco D (2020) Educational Technology Professional

Development in Higher Education: A

Systematic Literature Review of

Empirical Research. Front. Educ. 5:35.

doi: 10.3389/feduc.2020.00035

\section{Educational Technology Professional Development in Higher Education: A Systematic Literature Review of Empirical Research}

\author{
Sylvie Lidolf* and Denis Pasco \\ School of Education, University Bourgogne Franche-Comté, Besançon, France
}

The purpose of this study was to present a systematic literature review of empirical research in the emerging field of faculty members' educational technology professional development (ETPD). The focus of faculty members' ETPD is to ensure pedagogically sound technology use in the classroom. Thirty-five articles were identified from a systematic literature investigation, and the results revealed three postures that faculty members may adopt to promote their ETPD: faculty-as-learner, faculty-as-designer, and faculty-as-researcher. These results may help inform stakeholders and policymakers regarding key markers of faculty members' ETPD based on empirical evidence, to promote a sustainable and scalable educational change.

Keywords: educational technology, professional development, higher education, review, faculty member

\section{INTRODUCTION}

The digitization of the world can be considered a total social fact (Mauss, 1990) because it involves a systemic rethinking of society. As schools are responsible for preparing the next generation for their lives after formal education, teachers are expected to develop students' technological literacy, defined as the way an individual understands technology at a level that enables her/him to effectively function in a modern technological society (Gamire et al., 2006). This new demand undoubtedly affects teacher education, and over the past decade the integration of technology into the teaching and learning process has become the focus of major educational initiatives and reform efforts in teacher education (US Department of Education, 1999; Drent and Meelissen, 2008; Agyei and Voogt, 2011; Education Services Australia, 2012; Tondeur et al., 2012). Faculty members at large (i.e., those who teach in higher education) are in charge of implementing these reforms and, as noted by Uerz et al. (2018), they serve as "role models" for pre-service teachers and provide "good examples" of technology integration in the classroom. However, according to several studies (e.g., Gronseth et al., 2010; Tondeur et al., 2012), few faculty members effectively integrate technology into their teaching, for reasons including (1) lack of access to technology (Dawson, 2008), (2) insufficient courses or workshops to provide teacher educators with information and communication technology (ICT) skills (Wentworth et al., 2009), (3) lack of knowledge on how to integrate technology in the classroom (Tondeur et al., 2012), and (4) lack of technical support (Matthew et al., 2002). In response, Bai and Lehman (2003) called for faculty members' professional development, and a recent area of research focuses on adopting technology into pedagogical practice (Tondeur et al., 2017; Phuong et al., 2018; Uerz et al., 2018). King (2002) observed that substantial research has been conducted in relation to the concept of "Educational Technology 
Professional Development" (ETPD), which focuses on teachers' professional development as "an essential component to ensure pedagogically sound technology use in the classroom" (p. 284). The purpose of this literature review was to present a comprehensive understanding of this emerging field of research in higher education.

In the research literature, ETPD in higher education can be understood via three main theoretical approaches, which are based on technology, innovation, or socio-cultural interactions, respectively. Within the technology-based approach, the TPACK (Technological, Pedagogical and Content Knowledge) framework (Koehler and Mishra, 2005a,b; Mishra and Koehler, 2006; Koehler et al., 2007; Harris et al., 2009) is one of the most popular (Archambault et al., 2010; Rienties et al., 2013; Baya'a and Daher, 2015; Mourlam, 2017; Reyes et al., 2017; Jaipal-Jamani et al., 2018). Built on Shulman $(1986,1987)$ Pedagogical Content Knowledge framework, technological pedagogical knowledge is defined as "knowledge of the existence, components, and capabilities of various technologies as they are used in teaching, and conversely, knowing how teaching might change as the result of using particular technologies" (Mishra and Koehler, 2006, p. 1028). Although several authors have pointed out the limitations of TPACK (Psycharis and Kalogeria, 2017; Reyes et al., 2017), there are four main reasons for its widespread use in ETPD research in higher education: (1) it prevents faculty members from what Archambault et al. (2010) termed an ineffective technocentric approach, as the TPACK framework "integrates technology with the domains of content and pedagogy rather than allowing technology to be taught in isolation" (Harris et al., 2009, p. 402); (2) it guides faculty TPACK development (Mourlam, 2017; Jaipal-Jamani et al., 2018); (3) it provides a shared goal for the team during implementation (i.e., highlighting the desired qualities of twenty-first century teachers), which determines faculty outlook toward educational change (Esterhuizen et al., 2013; Becuwe et al., 2017; Reyes et al., 2017); and (4) it helps measure faculty TPACK level and development as an assessment instrument to evaluate ETPD effectiveness (e.g., Baya'a and Daher, 2015; Mourlam, 2017). When using the TPACK model, ETPD research in higher education found the following main results: (1) the development of TPACK knowledge and skills increased ICT proficiency and self-esteem (e.g., Baya'a and Daher, 2015; Mourlam, 2017), (2) perceived change of role (Archambault et al., 2010; Jaipal-Jamani et al., 2018), and (3) transformations in pedagogies (Archambault et al., 2010). In addition to the TPACK model, some researchers have used other frameworks within the technology-based approach. For instance, Chen et al. (2018), Christ et al. (2017), Dolk et al. (2002), and Triggs and John (2004) based their investigations on Vygotsky (1978) theory, looking to technology as an artifact and tool that mediates faculty professional development. Other researchers focused on the process of technology adoption using (1) the four progressive stages (substitution, augmentation, modification, redefinition)

\footnotetext{
Abbreviations: ETPD, educational technology professional development; ICT, information and communication technology; TPACK, technological pedagogical content knowledge.
}

of Puentedura (2010) SAMR (Substitution, Augmentation, Modification, Redefinition) model of technology integration (Psiropoulos et al., 2016), (2) the three levels (entry, adaptation, and transformation) of Coughlin and Lemke (1999) model (Seels et al., 2003), (3) the four stages (emerging, applying, infusing, transforming) of Anderson and van Weert's [(UNESCO, 2002)] model (Esterhuizen et al., 2013), or (4) the technology acceptance model (Ulrich and Karvonen, 2011) grounded in "perceived ease of use" and "perceived usefulness" factors as direct predictors of "intent to accept technology" (Davis, 1989; Venkatesh and Brown, 2001). Finally, Psycharis and Kalogeria (2017) investigated ETPD in higher education using a combination of several technology-based theoretical frameworks.

Within the innovation-based approach (Rogers, 1995; Teclehaimanot and Lamb, 2005; Maor, 2006; Drent and Meelissen, 2008; García and Roblin, 2008; Keengwe et al., 2009; King and Boyatt, 2014; Sher et al., 2015; Baran, 2016; Avidov-Ungar and Forkosh-Baruch, 2018) is one of the widely used to investigate ETPD in higher education (e.g., Keengwe et al., 2009; Baran, 2016). Rogers (1995) defined "innovativeness" as "the degree to which an individual or other unit of adoption is relatively earlier in adopting new ideas than other members of a system" (p. 252), while "innovation adoption" is "a decision to make full use of an innovation as the best course of action" (p. 36). Rogers (1995) identified five stages within the innovation adoption process (knowledge, persuasion, decision, implementation, confirmation) and five standardized "adopter categories" (innovator, early adopter, early majority, late majority, laggards) based on individuals' "socioeconomic status, personality values, and communication behavior" (p. 268), which enable measurement of their "innovativeness." Teclehaimanot and Lamb (2005) found useful Agarwal and Prasad (1998) tool to measure personal traits of innovativeness in the domain of information technology, as "a positive attitude toward any innovation increases the likelihood of the adoption of the innovation" (p. 3). Baran (2016) pointed out the fundamental role of peer-to-peer relationships and interpersonal channels in spreading innovation. In addition to Rogers' Diffusion of Innovations Theory, some researchers have used other frameworks within the innovation-based approach. For instance, Maor (2006) adopted Hagner and Schneebeck (2001) classification of information and communication technology adopters (entrepreneurs and risk takers, risk aversives, reward seekers, and reluctants) while King and Boyatt (2014) based their investigation on Russell (2009), which suggested that "innovations need to be woven into organizational context and centrally coordinated" (p. 1274). Even if some authors found different roles of "innovation" and used it at different stages of ETPD (e.g., Triggs and John, 2004; Drent and Meelissen, 2008; García and Roblin, 2008; Becuwe et al., 2017), they refer to innovation as a type of pedagogy that fits twenty-first century expectations for education. As Avidov-Ungar and ForkoshBaruch (2018) noted, "innovative pedagogy is needed as a means to actualize the vision to adapt the education system to the twenty-first century," with the ultimate goal of developing "high order thinking skills, creativity and self-learning, facilitating personal growth and social involvement, while strengthening 
the teacher as an educational leader of novel pedagogy" (p. 184). Finally, some authors investigated ETPD in higher education using a combination of innovation-based theoretical frameworks (e.g., Keengwe et al., 2009; Ulrich and Karvonen, 2011).

Finally, ETPD in higher education has also been investigated through a socio-cultural interactions-based approach referring to constructivist theories rooted in "the idea that learning is a social and collaborative process where cognitive development is possible through 'social interaction,' 'collaboration,' 'mentoring,' and 'exploration' (Piaget, 1972; Bruner, 1975; Vygotsky, 1978)" (Psiropoulos et al., 2016, p. 211). Within this approach, researchers examined interactions through different scales: at an individual scale (e.g., Sher et al., 2015; Jorgensen et al., 2018), at the "one-on-one" or "mentoring" scale (e.g., Matthew et al., 2002), and at the community scale (e.g., Shattuck and Anderson, 2013). For instance, at the individual scale, Sher et al. (2015) questioned individual perceived reasons for reluctance to engage in online education while Jorgensen et al. (2018) linked demographic data about professors who were nominated by their students for excellence in using technology, with their perceived proficiency, individual ICT experiences, and use. At the "one-on-one" or "mentoring" scale, Matthew et al. (2002) modeled a one-on-one technology coaching professional development project for teacher educators. Finally, the community scale is mostly based on Lave and Wenger's "communities of practice" or "communities of learners" (Lave, 1988; Lave and Wenger, 1991; Wenger, 1998; Wenger et al., 2002; Wenger-Trayner and Wenger-Trayner, 2015). Shattuck and Anderson (2013) demonstrated that there are several archetypes of "communities of practice," proposing an overview of identified networks of practice during their research: formal and informal, as well as internal (peers' community) and external (at workplaces/ in professional organizations) communities. Some research have investigated ETPD in higher education using different scales. For example, García and Roblin (2008) emphasized the modeled process from individual reflection to collaborative knowledge construction. Ashton and Newman (2006) "geographic hubs" described knowledge creation and transfer in terms of a cycle from an individual to small groups nested within increasingly larger groups. Chen et al. (2018) crossed "one-on-one" and community scales when examining the orientation and development of strategies or mediating tools used in the mentoring practice of a mathematics teacher educator-researcher and analyzing relationships between three levels of interaction (teacher educator-researcher, teachers, and students). Finally, in their attempt to provide empirical evidence of the effectiveness of an ETPD program for postdoctoral scholars in the STEM (Science, Technology, Engineering, and Mathematics) disciplines, Derting et al. (2016) used the three scales simultaneously. Biology postdoctoral scholars' perceptions of teaching strategies and of environmental factors influencing teaching were investigated individually (individual scale), regional team leaders served as long-distance teaching mentors (one-on-one scale), and teams of postdocs were created to co-design an entire learner-centered course and to reflect and discuss the challenges they encountered during their teaching experiences (community scale).
This article is the first attempt to conduct, from these three identified theoretical lenses, a systematic literature review on the emerging field of research of ETPD in higher education. The method section presents the article selection process, the findings reveal three main faculty members' postures as they deal with their ETPD, and the conclusion section highlights some key insights and suggests some prospects for future inquiry.

\section{METHODS}

\section{Search and Selection Criteria}

A systematic review of ETPD in higher education was conducted in April 2018 in major scholarly databases in educational research: Web of Science, Elsevier Science Direct, Springer, and Taylor \& Francis Group. To be considered for inclusion, articles had to meet the following criteria (1) empirical study, (2) peer-reviewed, data-based research articles published in English, (3) focused on faculty members ETPD in higher education. In the search query, three sets of search terms related to the topic were combined: ("teacher educator*" OR "teacher trainer*" OR "college instructor*" OR facult* OR lecturer* OR "higher education") AND ("professional development" OR development* OR training OR "educational change" OR transformation*) AND (technolog* OR ICT OR TPACK OR digital)

\section{Summarizing Articles and Analysis}

The search and selection process yielded a total of 35 articles that covered the three sets of search terms and met the aforementioned inclusion criteria (see Figure 1).

While reading the full-text versions of the articles, the following relevant data were extracted for inclusion in a table to allow a synthetic comparison (see Table 1):

1. General information about selected articles: authors, publication year, and country

2. Research design \& data analysis methods

3. Population: number of respondents (teacher educators or faculty members in higher education)

4. Educational context

5. Aim(s) of the research

6. Nature of data: data sources and investigated variables

7. Main characteristics of the ETPD project or program

8. Identified potential markers of ETPD

\section{RESULTS}

Three main categories emerged from cross-analysis. In reference to Lameul (2008), we refer to them as three kinds of postures that faculty members can adopt when engaged in ETPD: faculty-as-learner, faculty-as-designer, and faculty-asresearcher. The concept of posture is understood as the result in action of complex interactions between individual internal dispositions (beliefs, attitudes, intentions...), individual external dimensions (behaviors, professional gestures, practice...) and environmental components, within a given professional situation 


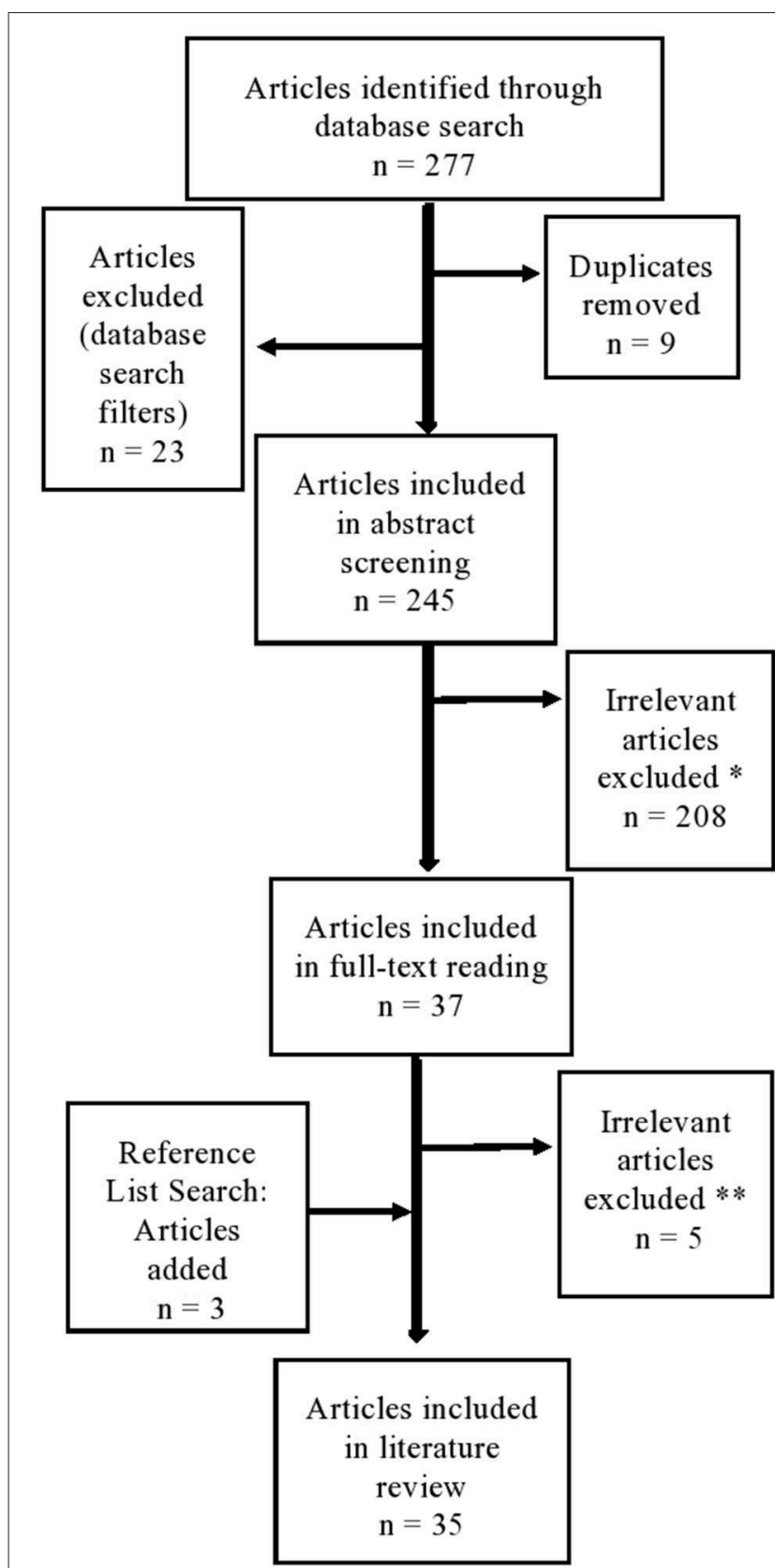

FIGURE 1 | Article search and selection process. *Reason for exclusion: not in higher education; ${ }^{* \star}$ Reason for exclusion: did not meet all eligible criteria.

(Lameul, 2008). Results are presented following these three kinds of postures.

\section{Faculty-As-Learner}

When centered on faculty members' learner posture, either at an individual scale, mentoring scale, or within a learner community, researchers have explored ways in which faculty members learn to integrate technology into teaching (Jorgensen et al., 2018), how faculty attitudes toward self-directed learning affect their use of technologies (Ulrich and Karvonen, 2011), or the limitations of technology self-teaching with respect to online teaching and learning (Sher et al., 2015). When it comes to faculty learning, some predominant characteristics regarding the most adequate learning approach have been identified.

Within the faculty-as-learner posture, learning is mainly understood as a developmental process rather than resulting from a transmission of knowledge. As Kukulska-Hulme (2012) noted, "the idea of training has morphed into development, which can be understood as an ongoing process concerned with changing attitudes and behaviors and preparing for the future" (p. 248). As a result, this changed the time perspective of the learning process, moving from time-limited periods of learning to lifelong learning, understood as the combination of processes throughout a lifetime (Kukulska-Hulme, 2012). For Seels et al. (2003), learning has even become a "way of life" (p. 92) and knowing how to learn is a new fundamental educational skill to acquire (Ashton and Newman, 2006). When positioning faculty as learners, researchers widely agree on the use of self-directed learning (Ulrich and Karvonen, 2011; Hoekstra and Crocker, 2015; Psiropoulos et al., 2016), which has been proven to be the distinguishing characteristic of efficient adult learning. Self-directed learning facilitates faculty engagement in the professional development process, in part because there is more personal responsibility on the learner side, which may lead faculty members to seek out professional ETPD opportunities within their professional practice. Moreover, Ashton and Newman (2006) argued that with heutagogy (i.e., a type of self-directed learning), learners' autonomy increases in proportion to teachers' withdrawal from the control process of knowledge transmission. Psiropoulos et al. (2016) presented a study based on a minimally invasive education (i.e., a radical type of self-directed learning), which demonstrated that technological knowledge and skills can be acquired serendipitously, intuitively, and creatively, when giving learners unrestricted access and adequate time to "play," explore, problem solve, and become critical thinkers, with almost no instruction (Mitra et al., 2005).

Some researchers have advanced the process of self-directed learning with faculty members by placing them in the position of learners within a learning context (Maor, 2006; KukulskaHulme, 2012; Esterhuizen et al., 2013; Psiropoulos et al., 2016). They argued that it is essential to give faculty members the opportunity to live an authentic and direct experience from a learner perspective, as this experience is considered a main source of learning and development in educational technology. For instance, Kukulska-Hulme (2012) used the "tactic of putting each faculty member in the position of online student" ( $p$. 248), while Psiropoulos et al. (2016) immersed faculty members in a new mobile teaching and learning environment, based on the implementation of iPads. The purpose of this strategy is for faculty members to personally experience as learners both the affordances and limitations of new technologies (e.g., Esterhuizen et al., 2013) or social constructivist pedagogies (e.g., Maor, 2006). Furthermore, Foley and Masingila (2014) stressed the importance of addressing all learners, insisting on the need to take learners' diversity into account and address a range of learning preferences. They found that the effective use of technology facilitated practices of universal design for 
TABLE 1 | Details of literature review articles.

\begin{tabular}{|c|c|c|c|c|c|c|c|}
\hline References & $\begin{array}{l}\text { Research design } \\
\text { (methods) }\end{array}$ & $\begin{array}{l}\text { Sample } \\
(n)\end{array}$ & $\begin{array}{l}\text { Educational } \\
\text { context }\end{array}$ & $\begin{array}{l}\text { Aim(s) } \\
\text { of research }\end{array}$ & $\begin{array}{l}\text { Data sources } \\
\text { (investigated variables) }\end{array}$ & $\begin{array}{l}\text { Main characteristics of ETPD } \\
\text { project/program }\end{array}$ & $\begin{array}{l}\text { Identified } \\
\text { ETPD markers }\end{array}$ \\
\hline $\begin{array}{l}\text { Archambault } \\
\text { et al. (2010) } \\
\text { (USA) }\end{array}$ & $\begin{array}{l}\text { Action Research } \\
\text { (AR), evaluative } \\
\text { (qualitative) }\end{array}$ & 20 & $\begin{array}{l}\text { College of } \\
\text { Teacher } \\
\text { Education and } \\
\text { Leadership }\end{array}$ & $\begin{array}{l}\text { To discuss the outcomes of an } \\
\text { ETPD project, the goal of which } \\
\text { was to transform pedagogy } \\
\text { integrating social-networking } \\
\text { tools }\end{array}$ & $\begin{array}{l}\text { Post-survey } \\
\text { (perceptions, TPACK) }\end{array}$ & $\begin{array}{l}\text { - Workshops: hands-on, } \\
\text { demonstrations, modeled uses } \\
\text { - Course unit re-design: } \\
\text { collaboration, communication, } \\
\text { problem solving } \\
\text { - Reflection } \\
\text { - Colleagues as resources: } \\
\text { wiki project }\end{array}$ & $\begin{array}{l}\text { - Positive impact on student } \\
\text { achievement } \\
\text { - Transformations in pedagogy } \\
\text { - Perceived change of role }\end{array}$ \\
\hline $\begin{array}{l}\text { Ashton and } \\
\text { Newman (2006) } \\
\text { (Australia) }\end{array}$ & $\begin{array}{l}\text { Exploratory } \\
\text { (mixed qualitative } \\
\text { data) }\end{array}$ & 8 & $\begin{array}{l}\text { School of } \\
\text { Education } \\
\text { (Early Childhood } \\
\text { department) }\end{array}$ & $\begin{array}{l}\text { To explore factors influencing } \\
\text { knowledge sharing in } \\
\text { communities of practice }\end{array}$ & $\begin{array}{l}\text { Questionnaires, phone calls, } \\
\text { focus groups (beliefs, use of } \\
\text { techno, perceptions, directors' } \\
\text { and employers' capabilities and } \\
\text { willingness to support employees } \\
\text { with ICT) }\end{array}$ & $\begin{array}{l}\text { - Questioning, research } \\
\text { - Reflection } \\
\text { - Communities of practice, geo } \\
\text { hub groups, linking profession } \\
\text { and university: collaboration } \\
\text { and shared discussion } \\
\text { - Lifelong learning } \\
\text { - Workshops: demonstration } \\
\text { and experimentation of ICT } \\
\text { uses } \\
\text { - Mapping } \\
\text { process: conceptualization }\end{array}$ & $\begin{array}{l}\text { - Introduction of innovative programs } \\
\text { using ICTs and heutagogy } \\
\text { - Diffusion of knowledge: production } \\
\text { of resources, portfolios } \\
\text { - Need to reframe pedagogy } \\
\text { - Tensions } \\
\text { - Role shift: from knowledge } \\
\text { transmitters to heutagogues } \\
\text { (knowledge brokers) }\end{array}$ \\
\hline $\begin{array}{l}\text { Avidov-Ungar } \\
\text { and } \\
\text { Forkosh-Baruch } \\
\text { (2018) (Israel) }\end{array}$ & $\begin{array}{l}\text { Phenomenography } \\
\text { (mixed) }\end{array}$ & & $\begin{array}{l}\text { Colleges of } \\
\text { Education (8) }\end{array}$ & $\begin{array}{l}\text { - To reflect on lived experiences } \\
\text { - To examine concept of "new } \\
\text { pedagogy" through teacher } \\
\text { educators' (TE) } \\
\text { professional identity }\end{array}$ & $\begin{array}{l}\text { Post-interviews (Perceptions on } \\
\text { pedagogy innovation) }\end{array}$ & $\begin{array}{l}\text { No ETPD project combined with } \\
\text { research project }\end{array}$ & $\begin{array}{l}\text { - Prof. identity: innovative TEs } \\
\text { - Changing role and teaching } \\
\text { approach } \\
\text { - New knowledge and competency: } \\
\text { to manage innovations and } \\
\text { change processes }\end{array}$ \\
\hline $\begin{array}{l}\text { Baran (2016) } \\
\text { (Turkey) }\end{array}$ & $\begin{array}{l}\text { Exploratory } \\
\text { (qualitative) }\end{array}$ & 12 pairs & Higher education & $\begin{array}{l}\text { To explore factors and critical } \\
\text { strategies for technology } \\
\text { adoption in practices } \\
\text { and to provide a faculty } \\
\text { technology mentoring model }\end{array}$ & $\begin{array}{l}\text { Blog posts, case reports, } \\
\text { interviews }\end{array}$ & $\begin{array}{l}\text { - Mentoring } \\
\text { - Workshops addressing needs, } \\
\text { exploring ICT affordances and } \\
\text { limitations, scaffolding, sharing } \\
\text { feedback, } \\
\text { TPACK development }\end{array}$ & $\begin{array}{l}\text { Motivation understood as "meeting } \\
\text { challenges" }\end{array}$ \\
\hline $\begin{array}{l}\text { Baya'a and } \\
\text { Daher (2015) } \\
\text { (Israel) }\end{array}$ & $\begin{array}{l}\text { Longitudinal, } \\
\text { evaluative } \\
\text { (quantitative) }\end{array}$ & 19 & $\begin{array}{l}\text { College of } \\
\text { Education }\end{array}$ & $\begin{array}{l}\text { - To accompany the } \\
\text { implementation of the College } \\
\text { ETPD intervention } \\
\text { - To examine instructors' } \\
\text { development of TPACK, } \\
\text { attitudes and proficiency }\end{array}$ & $\begin{array}{l}\text { Pre-post questionnaires (ICT } \\
\text { attitudes, ICT proficiency, TPACK } \\
\text { level) }\end{array}$ & $\begin{array}{l}\text { - Workshop: } 2 \text { levels, examples } \\
\text { and models of uses, exposure } \\
\text { to new pedagogy, design, ICT } \\
\text { support } \\
\text { - Factors of change (ranked by } \\
\text { TEs): workshop participation, } \\
\text { support, assistants' availability, } \\
\text { college policy, infrastructure }\end{array}$ & $\begin{array}{l}\text { - Developmentof TPACK, attitudes, } \\
\text { ICT proficiency (indicator of intention } \\
\text { of use) } \\
\text { - Self-esteem } \\
\text { - Increased number of web-based } \\
\text { learning environments, courses on } \\
\text { Moodle and ICT } \\
\text { pedagogical initiatives }\end{array}$ \\
\hline
\end{tabular}


TABLE 1 | Continued

\begin{tabular}{|c|c|c|c|c|c|c|c|}
\hline References & $\begin{array}{l}\text { Research design } \\
\text { (methods) }\end{array}$ & $\begin{array}{l}\text { Sample } \\
(n)\end{array}$ & $\begin{array}{l}\text { Educational } \\
\text { context }\end{array}$ & $\begin{array}{l}\text { Aim(s) } \\
\text { of research }\end{array}$ & $\begin{array}{l}\text { Data sources } \\
\text { (investigated variables) }\end{array}$ & $\begin{array}{l}\text { Main characteristics of ETPD } \\
\text { project/program }\end{array}$ & $\begin{array}{l}\text { Identified } \\
\text { ETPD markers }\end{array}$ \\
\hline $\begin{array}{l}\text { Becuwe et al. } \\
\text { (2017) } \\
\text { (Belgium, USA, } \\
\text { Netherlands) }\end{array}$ & $\begin{array}{l}\text { Delphi Study } \\
\text { (mixed) }\end{array}$ & 36 & $\begin{array}{l}\text { Teacher } \\
\text { Education } \\
\text { Institutions (4) }\end{array}$ & $\begin{array}{l}\text { To find consensual crucial } \\
\text { conditions for successful } \\
\text { implementation of TE Design } \\
\text { Teams for ICT integration }\end{array}$ & Literature review, questionnaires & No combined ETPD project & \\
\hline $\begin{array}{l}\text { Chen et al. } \\
\text { (2018) } \\
\text { (Taiwan) }\end{array}$ & $\begin{array}{l}\text { AR, longitudinal } \\
\text { (qualitative) }\end{array}$ & 1 & $\begin{array}{l}\text { University of } \\
\text { Technology and } \\
\text { Middle Schools } \\
\text { (2) }\end{array}$ & $\begin{array}{l}\text { To document process of } \\
\text { evolution of the continuous } \\
\text { design and revision of tools of a } \\
\text { Mathematic TE-Researcher } \\
\text { (MTE-R) }\end{array}$ & $\begin{array}{l}\text { Video, audio recordings, e-mails, } \\
\text { observations, reflective reports, } \\
\text { external funding organization's } \\
\text { report }\end{array}$ & $\begin{array}{l}\text { - Design-based ETPD } \\
\text { workshops } \\
\text { - Mentoring } \\
\text { - Reflective practitioner: } \\
\text { reflection on students' } \\
\text { performance and on } \\
\text { own practice }\end{array}$ & $\begin{array}{l}\text { - Identity: TE's professional growth } \\
\text { - Students' (= teachers) performance } \\
\text { - Reciprocal expansive } \\
\text { transformations of MTE-R's and } \\
\text { teachers' activity Tensions between } \\
\text { and within activity systems }\end{array}$ \\
\hline $\begin{array}{l}\text { Christ et al. } \\
\text { (2017) } \\
\text { (USA) }\end{array}$ & $\begin{array}{l}\text { Exploratory } \\
\text { (quantitative) }\end{array}$ & 208 & $\begin{array}{l}\text { Teacher } \\
\text { education } \\
\text { courses (977) }\end{array}$ & $\begin{array}{l}\text { - To explore TEs' video uses } \\
\text { - To identify relations between } \\
\text { variables and types of } \\
\text { video use }\end{array}$ & $\begin{array}{l}\text { Literature review, survey } \\
\text { (demographic, disciplinary, } \\
\text { beliefs, video properties) }\end{array}$ & No combined ETPD project & $\begin{array}{l}\text { - Increase of video use in TEs' } \\
\text { courses } \\
\text { - Use of various video methods } \\
\text { - Creation of one's own } \\
\text { case-study videos }\end{array}$ \\
\hline $\begin{array}{l}\text { Derting et al. } \\
\text { (2016) } \\
\text { (USA) }\end{array}$ & $\begin{array}{l}\text { Team Design } \\
\text { Based Research } \\
\text { (DBR), evaluative } \\
\text { (mixed) }\end{array}$ & 20 pairs & $\begin{array}{l}\text { Research, } \\
\text { comprehensive, } \\
\text { liberal arts, and } \\
\text { Community } \\
\text { Colleges }\end{array}$ & $\begin{array}{l}\text { - To test the effectiveness of the } \\
\text { ETPD program } \\
\text { - To provide evidence of impacts }\end{array}$ & $\begin{array}{l}\text { Questionnaires and surveys } \\
\text { (perceptions of teaching } \\
\text { strategies, environmental factors } \\
\text { influencing teaching, } \\
\text { self-efficacy), observations }\end{array}$ & $\begin{array}{l}\text { - Teams of postdoc } \\
\text { - Design, experience, reflection, } \\
\text { discussion } \\
\text { - Use of videos } \\
\text { - Long-distance teaching } \\
\text { mentors, immediate support } \\
\text { - Workshop }\end{array}$ & $\begin{array}{l}\text { - Enthusiasm } \\
\text { - Self-efficacy } \\
\text { - More student-centered pedagogy, } \\
\text { "reformed teaching" } \\
\text { - Need: evidence of long-term } \\
\text { transfer, longitudinal studies, } \\
\text { objective measures of student } \\
\text { learning and skills }\end{array}$ \\
\hline $\begin{array}{l}\text { Dolk et al. (2002) } \\
\text { (Netherlands) }\end{array}$ & $\begin{array}{l}\text { DBR, case study } \\
\text { (retrospective } \\
\text { analysis) }\end{array}$ & - & $\begin{array}{l}\text { In-service } \\
\text { course for } \\
\text { primary-school } \\
\text { mathematic TEs }\end{array}$ & To describe a TEs' ETPD initiative & $\begin{array}{l}\text { Field notes, interviews, } \\
\text { participants' written work, } \\
\text { meeting notes of researchers }\end{array}$ & $\begin{array}{l}\text { - Design, trial, revision of course } \\
\text { - Construction of knowledge } \\
\text { from practice } \\
\text { - Observing, questioning } \\
\text { observations, sharing and } \\
\text { discussing observations } \\
\text { (paraphrasing, role-playing), } \\
\text { reflecting, generalizing, } \\
\text { theorizing: ongoing } \\
\text { experimentation process }\end{array}$ & $\begin{array}{l}\text { - Narrative knowledge competency } \\
\text { - Expanding the personal repertoire } \\
\text { and generalizing to build a personal } \\
\text { didactical theory }\end{array}$ \\
\hline
\end{tabular}


TABLE 1 | Continued

\begin{tabular}{|c|c|c|c|c|c|c|c|}
\hline References & $\begin{array}{l}\text { Research design } \\
\text { (methods) }\end{array}$ & $\begin{array}{l}\text { Sample } \\
(n)\end{array}$ & $\begin{array}{l}\text { Educational } \\
\text { context }\end{array}$ & $\begin{array}{l}\text { Aim(s) } \\
\text { of research }\end{array}$ & $\begin{array}{l}\text { Data sources } \\
\text { (investigated variables) }\end{array}$ & $\begin{array}{l}\text { Main characteristics of ETPD } \\
\text { project/program }\end{array}$ & $\begin{array}{l}\text { Identified } \\
\text { ETPD markers }\end{array}$ \\
\hline $\begin{array}{l}\text { Drent and } \\
\text { Meelissen (2008) } \\
\text { (Netherlands) }\end{array}$ & $\begin{array}{l}\text { Exploratory, case } \\
\text { studies } \\
\text { (mixed) }\end{array}$ & 210 & $\begin{array}{l}\text { Teacher } \\
\text { Education } \\
\text { Institutes for } \\
\text { primary school } \\
\text { teachers }\end{array}$ & $\begin{array}{l}\text { Which factors stimulate or limit } \\
\text { innovative use of ICT by TEs? }\end{array}$ & $\begin{array}{l}\text { Literature review, national } \\
\text { questionnaire, interviews }\end{array}$ & $\begin{array}{l}\text { - No combined ETPD project } \\
\text { - Supportive conditions for } \\
\text { personal entrepreneurship: } \\
\text { - Development of cooperative } \\
\text { communities } \\
\text { - Stimulation of reflective } \\
\text { behavior } \\
\text { - Creation of facilities to } \\
\text { experiment with innovations }\end{array}$ & $\begin{array}{l}\text { - TE's profile with innovative use of } \\
\text { ICT: personal entrepreneurship (key } \\
\text { factor) } \\
\text { - Positive ICT attitude and perceived } \\
\text { change } \\
\text { - Student-oriented pedagogy } \\
\text { - ICT competency complies } \\
\text { with pedagogy }\end{array}$ \\
\hline $\begin{array}{l}\text { Esterhuizen et al. } \\
\text { (2013) } \\
\text { (South Africa) }\end{array}$ & $\begin{array}{l}\text { Exploratory, case } \\
\text { study, evaluative } \\
\text { (mixed) }\end{array}$ & 21 & $\begin{array}{l}\text { School of } \\
\text { Continuing } \\
\text { Teacher } \\
\text { Education } \\
\text { (University) }\end{array}$ & $\begin{array}{l}\text { - To experiment with a model for } \\
\text { faculty development toward } \\
\text { transformative learning } \\
\text { technology integration for } \\
\text { Open Distance Learning (ODL) } \\
\text { - To identify factors affecting } \\
\text { ODL adoption }\end{array}$ & $\begin{array}{l}\text { Literature review, questionnaire, } \\
\text { interviews (perceptions), } \\
\text { longitudinal observations }\end{array}$ & $\begin{array}{l}\text { - Organic, progressive-adaptive } \\
\text { training rather than } \\
\text { mechanistic operating } \\
\text { procedures } \\
\text { - Authentic learning tasks } \\
\text { - Learning by design } \\
\text { - TPACK training } \\
\text { - Collaboration }\end{array}$ & $\begin{array}{l}\text { - Changed roles } \\
\text { - Changed teaching and learning } \\
\text { approach: from instructivist to } \\
\text { constructivist } \\
\text { - Curriculum transformation } \\
\text { - Techno confidence }\end{array}$ \\
\hline $\begin{array}{l}\text { Foley and } \\
\text { Masingila (2014) } \\
\text { (USA, Kenya) }\end{array}$ & DBR (qualitative) & 21 & $\begin{array}{l}\text { Schools of } \\
\text { Education }\end{array}$ & $\begin{array}{l}\text { To provide ETPD to improve use } \\
\text { of technology in instruction } \\
\text { focused on Large Class } \\
\text { Pedagogy }\end{array}$ & $\begin{array}{l}\text { Survey (class size and strategies } \\
\text { for teaching large classes), } \\
\text { observations }\end{array}$ & $\begin{array}{l}\text { - Workshops: presentations and } \\
\text { hands-on exploration, } \\
\text { technology in teaching, in } \\
\text { scholarship, creation of online } \\
\text { identity, design } \\
\text { - Universal Design for Learning: } \\
\text { multiple means to access } \\
\text { course content, of action, of } \\
\text { expression and of } \\
\text { engagement, for students to } \\
\text { demonstrate } \\
\text { their competencies }\end{array}$ & $\begin{array}{l}\text { - Expanded use of course } \\
\text { management systems (Moodle) } \\
\text { - Mobile devices used as teaching } \\
\text { tools } \\
\text { - Creation of an online identity: } \\
\text { international connection } \\
\text { with scholars }\end{array}$ \\
\hline $\begin{array}{l}\text { Friel et al. (2009) } \\
\text { (USA) }\end{array}$ & $\begin{array}{l}\text { Experimented } \\
\text { model of ETPD, } \\
\text { descriptive }\end{array}$ & 59 & $\begin{array}{l}\text { College of } \\
\text { Business } \\
\text { Administration } \\
\text { (University) }\end{array}$ & $\begin{array}{l}\text { - To provide technology training } \\
\text { through pedagogical dialogue: } \\
\text { collaborative training team } \\
\text { - To understand factors for } \\
\text { technology integration and } \\
\text { faculty skill development }\end{array}$ & Pre-post surveys (perceptions) & $\begin{array}{l}\text { - Demonstrating and hands-on } \\
\text { experiences via constructivist } \\
\text { pedagogy } \\
\text { - Diverse ways of learning, } \\
\text { active learning techniques } \\
\text { - Pedagogical dialogue, } \\
\text { discussion about faculty fears } \\
\text { - Design, implementation, peers' } \\
\text { feedback, reflection } \\
\text { - Elaborate concepts } \\
\text { - Team } \\
\text { - Shared vision } \\
\text { - One-to-one faculty training } \\
\text { - Emphasize time on task }\end{array}$ & $\begin{array}{l}\text { - Role of ICT representatives } \\
\text { - Constructivist pedagogy } \\
\text { - Transformational change in practice } \\
\text { when there is alignment of physical } \\
\text { space redesign, technology training, } \\
\text { and pedagogical redesign } \\
\text { - Personal techno skills } \\
\text { - New skill: how to learn (continued } \\
\text { faculty development) }\end{array}$ \\
\hline
\end{tabular}


TABLE 1 | Continued

\begin{tabular}{|c|c|c|c|c|c|c|c|}
\hline References & $\begin{array}{l}\text { Research design } \\
\text { (methods) }\end{array}$ & $\begin{array}{l}\text { Sample } \\
(n)\end{array}$ & $\begin{array}{l}\text { Educational } \\
\text { context }\end{array}$ & $\begin{array}{l}\operatorname{Aim}(\mathbf{s}) \\
\text { of research }\end{array}$ & $\begin{array}{l}\text { Data sources } \\
\text { (investigated variables) }\end{array}$ & $\begin{array}{l}\text { Main characteristics of ETPD } \\
\text { project/program }\end{array}$ & $\begin{array}{l}\text { Identified } \\
\text { ETPD markers }\end{array}$ \\
\hline $\begin{array}{l}\text { García and } \\
\text { Roblin (2008) } \\
\text { (Spain) }\end{array}$ & AR (qualitative) & 5 & $\begin{array}{l}\text { University } \\
\text { (psycho- } \\
\text { pedagogy } \\
\text { faculty) }\end{array}$ & $\begin{array}{l}\text { To study dialectic interaction } \\
\text { amongst innovation, research, } \\
\text { and ETPD processes }\end{array}$ & $\begin{array}{l}\text { Observation, interviews, focus } \\
\text { groups, student self-assessment } \\
\text { and short stories, reflective blogs }\end{array}$ & $\begin{array}{l}\text { - Reflection, dialogue, } \\
\text { collaboration, knowledge } \\
\text { construction } \\
\text { - Diversity: teaching styles } \\
\text { - Inquiry stance } \\
\text { - Team: group cohesion (shared } \\
\text { goals) } \\
\text { - Connections between class } \\
\text { contents and real-life } \\
\text { experiences } \\
\text { - Learning climate and } \\
\text { affective dimensions }\end{array}$ & $\begin{array}{l}\text { - Criterion of success: strong and } \\
\text { authentic feeling of transformation in } \\
\text { one's practice } \\
\text { - Evolutions: from: Initial expectation } \\
\text { to receptiveness and appropriation } \\
\text { of project/tools } \\
\text { - Evaluation: seen as control \& } \\
\text { grading/then, as a continuous } \\
\text { process of learning \& a shared } \\
\text { commitment/responsibility, } \\
\text { self-evaluation \& self-grading } \\
\text { - Motivation: from active involvement } \\
\text { to a sense of shared responsibility } \\
\text { - Awareness of students' interests, } \\
\text { motivations, needs } \\
\text { - Role of Ph-D student } \\
\text { - Away from fear of losing identity or } \\
\text { control } \\
\text { - Expansion of learning beyond } \\
\text { the classroom }\end{array}$ \\
\hline $\begin{array}{l}\text { Hoekstra and } \\
\text { Crocker (2015) } \\
\text { (Canada) }\end{array}$ & $\begin{array}{l}\text { DBR, evaluative } \\
\text { (mixed) }\end{array}$ & 102 & $\begin{array}{l}\text { Post-secondary } \\
\text { vocational } \\
\text { education } \\
\text { institute (13 } \\
\text { departments) }\end{array}$ & $\begin{array}{l}\text { To determine adoption, reception } \\
\text { by faculty of an e-portfolio } \\
\text { approach and identification of its } \\
\text { impact on faculty ETPD }\end{array}$ & $\begin{array}{l}\text { Literature review, focus groups } \\
\text { (aspects of faculty role and } \\
\text { sources of evidence of } \\
\text { performance), post-survey and } \\
\text { interview (adoption, perceived } \\
\text { impact of e-portfolio approach) }\end{array}$ & $\begin{array}{l}\text { - Reflection } \\
\text { - Sharing: learning communities } \\
\text { - Design: feedback tools, PD } \\
\text { plans, goals, to be discussed } \\
\text { with supervisors } \\
\text { - Workshops } \\
\text { - Mentoring } \\
\text { - Collaboration }\end{array}$ & $\begin{array}{l}\text { - Motivation: from mandatory to } \\
\text { voluntary nature ofe-portfolio } \\
\text { (reception/ interest) } \\
\text { - Increased awareness of areas of } \\
\text { improvement } \\
\text { - More and different explicit sources } \\
\text { of feedback on several aspects of } \\
\text { own role }\end{array}$ \\
\hline $\begin{array}{l}\text { Jaipal-Jamani } \\
\text { et al. (2018) } \\
\text { (Canada, USA) }\end{array}$ & $\begin{array}{l}\text { DBR and TPACK } \\
\text { Professional } \\
\text { Learning Design } \\
\text { (TPLD) Model, } \\
\text { case study } \\
\text { (qualitative) }\end{array}$ & 4 & $\begin{array}{l}\text { Faculty of } \\
\text { education }\end{array}$ & $\begin{array}{l}\text { To examine how faculty taking on } \\
\text { technology leadership roles } \\
\text { develop TPACK knowledge and } \\
\text { build capacity for } \\
\text { techno-enhanced teaching }\end{array}$ & $\begin{array}{l}\text { Pre-post interviews, field notes, } \\
\text { observations, video, artifacts }\end{array}$ & $\begin{array}{l}\text { - Reflexive, self-study } \\
\text { - Learning communities } \\
\text { - Size: small group working in } \\
\text { same course content } \\
\text { or discipline }\end{array}$ & $\begin{array}{l}\text { - Roles: from learner to leader } \\
\text { - Role of leadership (workshop } \\
\text { facilitator): improved knowledge } \\
\text { and competencies in } \\
\text { technology-enhanced teaching } \\
\text { - Comfort level in adopting new } \\
\text { teaching methods impacted the } \\
\text { way of using the TPLD model }\end{array}$ \\
\hline
\end{tabular}


TABLE 1 | Continued

\begin{tabular}{|c|c|c|c|c|c|c|c|}
\hline References & $\begin{array}{l}\text { Research design } \\
\text { (methods) }\end{array}$ & $\begin{array}{l}\text { Sample } \\
(n)\end{array}$ & $\begin{array}{l}\text { Educational } \\
\text { context }\end{array}$ & $\begin{array}{l}\text { Aim(s) } \\
\text { of research }\end{array}$ & $\begin{array}{l}\text { Data sources } \\
\text { (investigated variables) }\end{array}$ & $\begin{array}{l}\text { Main characteristics of ETPD } \\
\text { project/program }\end{array}$ & $\begin{array}{l}\text { Identified } \\
\text { ETPD markers }\end{array}$ \\
\hline $\begin{array}{l}\text { Jorgensen et al. } \\
\text { (2018) (Canada) }\end{array}$ & $\begin{array}{l}\text { Exploratory, } \\
\text { descriptive } \\
\text { (mixed) }\end{array}$ & 114 & $\begin{array}{l}\text { Urban junior } \\
\text { College, } \\
\text { Community } \\
\text { College }\end{array}$ & $\begin{array}{l}\text { To explore technology related } \\
\text { pedagogical practices of college } \\
\text { professors nominated by their } \\
\text { students to be excellent in using } \\
\text { technology in teaching }\end{array}$ & $\begin{array}{l}\text { Questionnaires and interviews } \\
\text { (demographic, perceived } \\
\text { proficiency, previous ICT } \\
\text { experiences, ICT used in } \\
\text { teaching checklist) }\end{array}$ & $\begin{array}{l}\text { - No combined ETPD project } \\
\text { - Collaboration for technology } \\
\text { problem solving } \\
\text { - ICT self-learning: trial and error } \\
\text { (back-up plans), peer and ICT } \\
\text { center support, online } \\
\text { resources, workshops } \\
\text { - Workshops: not practical } \\
\text { enough in the content, too } \\
\text { basic, rare or not scheduled at } \\
\text { convenient times }\end{array}$ & $\begin{array}{l}\text { - Students' nomination of TEs as } \\
\text { excellent in using techno in their } \\
\text { teaching } \\
\text { - Used techno } \\
\text { - Perceived proficiency }\end{array}$ \\
\hline $\begin{array}{l}\text { Keengwe et al. } \\
\text { (2009) (USA) }\end{array}$ & $\begin{array}{l}\text { Exploratory } \\
\text { (qualitative) }\end{array}$ & 25 & Public University & $\begin{array}{l}\text { To explore factors affecting ICT } \\
\text { adoption }\end{array}$ & Narratives & $\begin{array}{l}\text { - No combined ETPD project } \\
\text { - Resources: examples, best } \\
\text { practices } \\
\text { - Collaboration } \\
\text { - Instructional design support } \\
\text { needed } \\
\text { - Workshops connected to } \\
\text { needs } \\
\text { - Communication and } \\
\text { awareness process }\end{array}$ & \\
\hline $\begin{array}{l}\text { King and Boyatt } \\
\text { (2014) } \\
\text { (United Kingdom) }\end{array}$ & $\begin{array}{l}\text { Phenomenological } \\
\text { approach } \\
\text { (qualitative) }\end{array}$ & 48 & University & $\begin{array}{l}\text { To explore factors influencing } \\
\text { adoption of e-learning }\end{array}$ & $\begin{array}{l}\text { Focus groups, individual } \\
\text { interviews (perceptions) }\end{array}$ & $\begin{array}{l}\text { - No combined ETPD project } \\
\text { - Diversity: varied program of } \\
\text { staff development } \\
\text { - Sharing practice } \\
\text { - On-going consultation and } \\
\text { collaboration with staff } \\
\text { - Communities of practice } \\
\text { - Workshops closely tailored to } \\
\text { specific staff needs, } \\
\text { pedagogic-driven, face-to-face } \\
\text { support and online guidance }\end{array}$ & $\begin{array}{l}\text { Changed attitudes, skills, and } \\
\text { confidence level }\end{array}$ \\
\hline
\end{tabular}




\begin{tabular}{|c|c|c|c|c|c|c|c|}
\hline References & $\begin{array}{l}\text { Research design } \\
\text { (methods) }\end{array}$ & $\begin{array}{l}\text { Sample } \\
(n)\end{array}$ & $\begin{array}{l}\text { Educational } \\
\text { context }\end{array}$ & $\begin{array}{l}\text { Aim(s) } \\
\text { of research }\end{array}$ & $\begin{array}{l}\text { Data sources } \\
\text { (investigated variables) }\end{array}$ & $\begin{array}{l}\text { Main characteristics of ETPD } \\
\text { project/program }\end{array}$ & $\begin{array}{l}\text { Identified } \\
\text { ETPD markers }\end{array}$ \\
\hline $\begin{array}{l}\text { Kukulska-Hulme } \\
\text { (2012) } \\
\text { (United Kingdom) }\end{array}$ & $\begin{array}{l}\text { Evaluative } \\
\text { (qualitative) }\end{array}$ & 24 & Open University & $\begin{array}{l}\text { - To reflect on ETPD experiences } \\
\text { - To propose a lifelong } \\
\text { learning perspective }\end{array}$ & $\begin{array}{l}\text { Interviews (feedback from staff } \\
\text { about the Mobile Learning } \\
\text { Guide), evaluation forms (event) }\end{array}$ & $\begin{array}{l}\text { - Experiencing being an online } \\
\text { student } \\
\text { - ETPD connected with } \\
\text { pedagogy and disciplinary } \\
\text { context } \\
\text { - Need for self-management, } \\
\text { self-awareness, meta-learning } \\
\text { - Community of practice } \\
\text { - Combine research and inquiry } \\
\text { with teaching and learning } \\
\text { - Learning community (informal) } \\
\text { vs. centrally organized } \\
\text { seminars and workshops } \\
\text { - Technology adoption for } \\
\text { professional learning beyond } \\
\text { use for teaching } \\
\text { - Collaborative training team; } \\
\text { co-development of faculty and } \\
\text { students } \\
\text { - Workshops: hands-on, } \\
\text { concrete experiences } \\
\text { addressing faculty needs; } \\
\text { preference for case studies } \\
\text { - One-on-one support }\end{array}$ & $\begin{array}{l}\text { From "training" to "development" = } \\
\text { an ongoing process concerned with } \\
\text { changing attitudes and behaviors and } \\
\text { more personal responsibility } \\
\text { - Role: "professional role model" } \\
\text { - Lifelong learner: continually } \\
\text { changing person } \\
\text { - Competencies: self-management, } \\
\text { self-awareness, meta-learning, } \\
\text { experience, and social interaction in } \\
\text { a community of practice } \\
\text { - Personal conviction }\end{array}$ \\
\hline $\begin{array}{l}\text { Maor (2006) } \\
\text { (Australia) }\end{array}$ & $\begin{array}{l}\text { AR, Longitudinal, } \\
\text { case studies } \\
\text { (qualitative) }\end{array}$ & 10 & University & $\begin{array}{l}\text { - To introduce social } \\
\text { constructivist pedagogy, } \\
\text { provide technical support, and } \\
\text { create a community of learners } \\
\text { - To increase interdisciplinary } \\
\text { collaboration and } \\
\text { investigate changes }\end{array}$ & $\begin{array}{l}\text { Pre-questionnaire } \\
\text { (teaching/teaching online) } \\
\text { experience, perception (and } \\
\text { reasons for use), diagrams and } \\
\text { interviews (perceptions) }\end{array}$ & $\begin{array}{l}\text { - Experiential model: } \\
\text { teacher-as-a-learner } \\
\text { - Teacher-as-a-researcher } \\
\text { - Reflective approach } \\
\text { - Communication: one of ETPD } \\
\text { goals: for problem- and } \\
\text { dilemma- solving } \\
\text { - Introduction to social } \\
\text { constructivist pedagogy } \\
\text { - Monthly ETPD workshops }\end{array}$ & $\begin{array}{l}\text { - Diagram: diagnostical and } \\
\text { developmental tool } \\
\text { - Dilemmas } \\
\text { - } 5 \text { types of approach to innovative } \\
\text { teaching with new technology }\end{array}$ \\
\hline $\begin{array}{l}\text { Mourlam (2017) } \\
\text { (USA) }\end{array}$ & DBR (mixed) & 5 & $\begin{array}{l}\text { Comprehensive } \\
\text { University }\end{array}$ & $\begin{array}{l}\text { To propose a model for faculty } \\
\text { TPACK development }\end{array}$ & $\begin{array}{l}\text { Pre-post self-report surveys } \\
\text { (TPACK), field notes, pre-post } \\
\text { semi-structured interviews } \\
\text { (TPACK) }\end{array}$ & $\begin{array}{l}3 \mathrm{~h} \text { kickoff workshop: addressing } \\
\text { needs, hands-on practice, } \\
\text { as-a-learner position, play } \\
\text { TPACK Game, design of } \\
\text { individual instruction project, } \\
\text { peer collaboration } \\
\text { - Evaluation with peers } \\
\text { - Ongoing and personalized } \\
\text { experience } \\
\text { - Developer support }\end{array}$ & $\begin{array}{l}\text { - Increased confidence and TPACK } \\
\text { - Increased TPACK for TES and } \\
\text { Teacher candidates } \\
\text { - Increased faculty instructional } \\
\text { risk taking }\end{array}$ \\
\hline
\end{tabular}


TABLE 1 | Continued

\begin{tabular}{|c|c|c|c|c|c|c|c|}
\hline References & $\begin{array}{l}\text { Research design } \\
\text { (methods) }\end{array}$ & $\begin{array}{l}\text { Sample } \\
\text { (n) }\end{array}$ & $\begin{array}{l}\text { Educational } \\
\text { context }\end{array}$ & $\begin{array}{l}\text { Aim(s) } \\
\text { of research }\end{array}$ & $\begin{array}{l}\text { Data sources } \\
\text { (investigated variables) }\end{array}$ & $\begin{array}{l}\text { Main characteristics of ETPD } \\
\text { project/program }\end{array}$ & $\begin{array}{l}\text { Identified } \\
\text { ETPD markers }\end{array}$ \\
\hline $\begin{array}{l}\text { Psiropoulos } \\
\text { et al. (2016) } \\
\text { (United Arab } \\
\text { Emirates) }\end{array}$ & $\begin{array}{l}\text { Longitudinal case } \\
\text { study, evaluative } \\
\text { (qualitative) }\end{array}$ & 16 & $\begin{array}{l}\text { Urban women's } \\
\text { college, higher } \\
\text { colleges of } \\
\text { technology }\end{array}$ & $\begin{array}{l}\text { To measure effectiveness of an } \\
\text { iPad PD program }\end{array}$ & $\begin{array}{l}\text { Literature review (ETPD } \\
\text { effectiveness), post-interviews, } \\
\text { participant observation, online } \\
\text { discussion forum }\end{array}$ & $\begin{array}{l}\text { - Communication } \\
\text { - Collaboration } \\
\text { - Reflection } \\
\text { - Learning community; multiple } \\
\text { learning options } \\
\text { - Design: situated, content } \\
\text { specific; co-design } \\
\text { - Workshops: individual needs } \\
\text { considered; conference; } \\
\text { display of range of practical } \\
\text { apps of mobile pedagogy from } \\
\text { external guest speaker (Apple) } \\
\text { - SAMR and/or TPACK: } \\
\text { guidance on transformative } \\
\text { pedagogy } \\
\text { - One-on-one online mentor }\end{array}$ & $\begin{array}{l}\text { - Shift of beliefs and perceptions } \\
\text { - Comfort level // lever for readiness } \\
\text { (to lead ETPD sessions or make } \\
\text { professional presentations) and } \\
\text { engagement; progression: from } \\
\text { basic operational level to pedagogy } \\
\text { implications and student success; } \\
\text { from passively receiving input } \\
\text { to actively sharing and giving } \\
\text { presentations } \\
\text { - Role: pedagogical partner } \\
\text { - Transformational theory: } 10 \text { phases } \\
\text { caused by a "disorienting dilemma" }\end{array}$ \\
\hline $\begin{array}{l}\text { Psycharis and } \\
\text { Kalogeria (2017) } \\
\text { (Greece) }\end{array}$ & $\begin{array}{l}\text { Longitudinal, study } \\
\text { cases (qualitative) }\end{array}$ & 16 & $\begin{array}{l}\text { University } \\
\text { Centers } \\
\text { (Mathematics } \\
\text { department) }\end{array}$ & $\begin{array}{l}\text { - To connect TPACK to TE's } \\
\text { epistemologies and practices } \\
\text { - To identify operational } \\
\text { invariants in Documentation } \\
\text { Work and factors influencing } \\
\text { teaching of TPACK }\end{array}$ & $\begin{array}{l}\text { Literature review, documentation } \\
\text { work, researchers' notes }\end{array}$ & $\begin{array}{l}\text { - Practicum: observation of TEs, } \\
\text { reflexivity, design, } \\
\text { implementation, reflexivity } \\
\text { - Diversity of practices } \\
\text { experienced } \\
\text { - Resources: generic scenarios, } \\
\text { teaching models } \\
\text { - Conceptualization of } \\
\text { experiences } \\
\text { - Narratives } \\
\text { - Acting "as students" } \\
\text { - Teaching preferences } \\
\text { - Mentor }\end{array}$ & $\begin{array}{l}\text { - Roles: explainer, instructor, facilitator } \\
\text { - Dilemmas } \\
\text { - Tools' evolution } \\
\text { (documentational genesis) }\end{array}$ \\
\hline $\begin{array}{l}\text { Reyes et al. } \\
\text { (2017) (Australia) }\end{array}$ & $\begin{array}{l}\text { Exploratory } \\
\text { (quantitative: } \\
\text { multivariate } \\
\text { statistical } \\
\text { technique-cluster } \\
\text { analysis) }\end{array}$ & 51 & $\begin{array}{l}\text { Regional } \\
\text { University }\end{array}$ & $\begin{array}{l}\text { - To explore TEs' uses of ICT } \\
\text { - To understand perceived } \\
\text { impact of TPACK in practices } \\
\text { of Tes }\end{array}$ & Survey, interviews (perceptions) & No combined ETPD project & \\
\hline
\end{tabular}


TABLE 1 | Continued

\begin{tabular}{|c|c|c|c|c|c|c|c|}
\hline References & $\begin{array}{l}\text { Research design } \\
\text { (methods) }\end{array}$ & $\begin{array}{l}\text { Sample } \\
(n)\end{array}$ & $\begin{array}{l}\text { Educational } \\
\text { context }\end{array}$ & $\begin{array}{l}\text { Aim(s) } \\
\text { of research }\end{array}$ & $\begin{array}{l}\text { Data sources } \\
\text { (investigated variables) }\end{array}$ & $\begin{array}{l}\text { Main characteristics of ETPD } \\
\text { project/program }\end{array}$ & $\begin{array}{l}\text { Identified } \\
\text { ETPD markers }\end{array}$ \\
\hline $\begin{array}{l}\text { Rienties et al. } \\
\text { (2013) } \\
\text { (United Kingdom, } \\
\text { Netherlands) }\end{array}$ & $\begin{array}{l}\text { Longitudinal, } \\
\text { evaluative } \\
\text { (quantitative) }\end{array}$ & 33 & $\begin{array}{l}\text { Higher } \\
\text { Educational } \\
\text { Institutions (3 } \\
\text { disciplines) }\end{array}$ & $\begin{array}{l}\text { - To measure effects of an online } \\
\text { PD } \\
\text { - To demonstrate its impact }\end{array}$ & $\begin{array}{l}\text { Pre-post questionnaires } \\
\text { (Teachers' beliefs and intentions, } \\
\text { TPACK) }\end{array}$ & $\begin{array}{l}\text { - Online modules: collaborative } \\
\text { knowledge building, } \\
\text { educational apps, measuring } \\
\text { knowledge and understanding, } \\
\text { supervising students in } \\
\text { distance learning } \\
\text { - Active learning environment: } \\
\text { small groups, peer } \\
\text { discussions, implementation of } \\
\text { redesigned module, reflection; } \\
\text { flexibility, time investment for } \\
\text { learners: } 20-25 \text { h over } \\
12 \text { weeks }\end{array}$ & $\begin{array}{l}\text { - TPACK knowledge and skills } \\
\text { increased } \\
\text { - Lower intentions toward knowledge } \\
\text { transmission } \\
\text { - Increased usage of technology and } \\
\text { confidence level in abilities }\end{array}$ \\
\hline $\begin{array}{l}\text { Rowe et al. } \\
\text { (2013) (South } \\
\text { Africa) }\end{array}$ & $\begin{array}{l}\text { Delphi study } \\
\text { (qualitative) }\end{array}$ & 25 & $\begin{array}{l}\text { Higher } \\
\text { Education } \\
\text { (Clinical } \\
\text { education) }\end{array}$ & $\begin{array}{l}\text { To identify professional attributes } \\
\text { beyond knowledge and skills, } \\
\text { and strategies to develop these } \\
\text { attributes }\end{array}$ & Surveys & $\begin{array}{l}\text { - No combined ETPD project } \\
\text { - Discussion and sharing of } \\
\text { personal values, resources, } \\
\text { and experiences } \\
\text { - Case studies, problem-based } \\
\text { learning } \\
\text { - Role modeling } \\
\text { - Active engagement with ethical } \\
\text { and emotional contexts } \\
\text { - Reflectiveness on stress, } \\
\text { emotions } \\
\text { - Access content outside the } \\
\text { classroom } \\
\text { - Diversity of teaching strategies } \\
\text { - Team: peer-supported small } \\
\text { groups } \\
\text { - Interprofessional collaboration }\end{array}$ & $\begin{array}{l}\text { - Positive attitudes toward continuing } \\
\text { personal and PD } \\
\text { - Self-directed } \\
\text { self-regulation } \\
\text { - Professional identity: professional } \\
\text { attributes beyond "having" } \\
\text { knowledge and skills } \\
\text { - From transmission models (rote } \\
\text { learning of knowledge and technical } \\
\text { skills) to facilitating pedagogy }\end{array}$ \\
\hline $\begin{array}{l}\text { Seels et al. } \\
\text { (2003) (USA) }\end{array}$ & $\begin{array}{l}\text { Formative } \\
\text { evaluation study, } \\
\text { case studies } \\
\text { (mixed) }\end{array}$ & 20 & $\begin{array}{l}\text { University, public } \\
\text { and private } \\
\text { schools, } \\
\text { non-profit, } \\
\text { corporate and } \\
\text { industry partners }\end{array}$ & $\begin{array}{l}\text { - To encourage technology } \\
\text { integration, support innovation, } \\
\text { adoption and resource sharing } \\
\text { by creating Collaborative } \\
\text { Communities of Learners } \\
\text { (CCOLs) } \\
\text { - To identify changes }\end{array}$ & $\begin{array}{l}\text { Event evaluations, reflective } \\
\text { notes, project checklists, video, } \\
\text { interviews, pre-post surveys } \\
\text { (technology skills, attitudes, } \\
\text { preferences, and interests), } \\
\text { course syllabi or artifacts, CCOLs } \\
\text { tool (trust, communication, } \\
\text { comfort level, overall feelings) }\end{array}$ & $\begin{array}{l}\text { - CCOLs: collaboration, } \\
\text { enhancement, enactment, } \\
\text { reflection; for effective, } \\
\text { sustaining CCOLs: } \\
\text { communication, common } \\
\text { goals, commitment, sharing, } \\
\text { and learning, interdependence } \\
\text { - Mentoring } \\
\text { - Workshops: } 3 \text { levels; new } \\
\text { technology with small } \\
\text { audiences; relevant, } \\
\text { individualized project }\end{array}$ & $\begin{array}{l}\text { - Changed attitudes, preferences, } \\
\text { skills } \\
\text { - New skills: frustration shooting, } \\
\text { grant writing } \\
\text { - Mentor: role; interpersonal skills: } \\
\text { experience with instruction and } \\
\text { flexibility to adjust to adopter } \\
\text { - Changed syllabi } \\
\text { - Increased use of technology }\end{array}$ \\
\hline
\end{tabular}


TABLE 1 | Continued

\begin{tabular}{|c|c|c|c|c|c|c|c|}
\hline References & $\begin{array}{l}\text { Research design } \\
\text { (methods) }\end{array}$ & $\begin{array}{l}\text { Sample } \\
(n)\end{array}$ & $\begin{array}{l}\text { Educational } \\
\text { context }\end{array}$ & $\begin{array}{l}\text { Aim(s) } \\
\text { of research }\end{array}$ & $\begin{array}{l}\text { Data sources } \\
\text { (investigated variables) }\end{array}$ & $\begin{array}{l}\text { Main characteristics of ETPD } \\
\text { project/program }\end{array}$ & $\begin{array}{l}\text { Identified } \\
\text { ETPD markers }\end{array}$ \\
\hline $\begin{array}{l}\text { Shattuck and } \\
\text { Anderson (2013) } \\
\text { (USA and } \\
\text { Canada) }\end{array}$ & $\begin{array}{l}\text { DBR, evaluative } \\
\text { (qualitative) }\end{array}$ & 24 & $\begin{array}{l}\text { Online training } \\
\text { course }\end{array}$ & $\begin{array}{l}\text { - To identify principles for training } \\
\text { instructors to teach online } \\
\text { - To evaluate impact of an online } \\
\text { training course on participants' } \\
\text { later practice }\end{array}$ & $\begin{array}{l}\text { Literature review (online teaching } \\
\text { roles and competencies), survey } \\
\text { and questionnaire, interviews } \\
\text { and virtual focus groups, } \\
\text { archived online courses }\end{array}$ & $\begin{array}{l}\text { - Experiencing being an online } \\
\text { student } \\
\text { - Reflecting on personal } \\
\text { teaching role as online } \\
\text { instructors } \\
\text { - Being part of a community of } \\
\text { learners } \\
\text { - Immersion in an online } \\
\text { environment living in a foreign } \\
\text { country: frustration, confusion, } \\
\text { self-doubt, fear, then rethinking } \\
\text { normal or commonplace } \\
\text { behaviors } \\
\text { - Re-designing online course } \\
\text { - Use of metaphors // abstract/ } \\
\text { conceptual level }\end{array}$ & $\begin{array}{l}\text { - Participants' use of new knowledge } \\
\text { and skills } \\
\text { - Role shift: facilitator } \\
\text { - Learning // process of enculturation; } \\
\text { "discombobulating" experience of } \\
\text { immersion } \\
\text { - Empathy with learners: more } \\
\text { student-centered perspective } \\
\text { - Rethinking of practice at least } \\
\text { - Use of metaphors }\end{array}$ \\
\hline $\begin{array}{l}\text { Sher et al. (2015) } \\
\text { (Australia) }\end{array}$ & $\begin{array}{l}\text { Exploratory, Lived } \\
\text { experience } \\
\text { research approach } \\
\text { (mixed) }\end{array}$ & $\begin{array}{l}54 \% \text { of all } \\
\text { full-time CM } \\
\text { academics in } \\
\text { Australia }\end{array}$ & $\begin{array}{l}\text { University } \\
\text { (Construction } \\
\text { Management } \\
\text { programs) }\end{array}$ & $\begin{array}{l}\text { To identify and understand } \\
\text { factors impacting } \mathrm{CM} \text { academics } \\
\text { activities }\end{array}$ & $\begin{array}{l}\text { Survey, interviews, focus groups } \\
\text { (perceived reasons for reticence } \\
\text { to online education) }\end{array}$ & $\begin{array}{l}\text { - No combined ETPD project } \\
\text { - Self-teaching: lack of online } \\
\text { course development and } \\
\text { delivery skills }\end{array}$ & $\begin{array}{l}\text { - Change of practice } \\
\text { - Away from fear of losing identity } \\
\text { built on university reputation of } \\
\text { providing strong } \\
\text { face-to-face experience) }\end{array}$ \\
\hline $\begin{array}{l}\text { Teclehaimanot } \\
\text { and Lamb } \\
\text { (2005) (USA) }\end{array}$ & Evaluative (survey) & 31 & $\begin{array}{l}\text { University } \\
\text { (College of } \\
\text { Education, } \\
\text { College of Arts } \\
\text { and Science } \\
\text { Faculty) }\end{array}$ & $\begin{array}{l}\text { - To assist TEs in integration of } \\
\text { technology into courses } \\
\text { - To describe evolution of this } \\
\text { program and identify ways to } \\
\text { enhance PD experience }\end{array}$ & $\begin{array}{l}\text { PD evaluative surveys (perceived } \\
\text { usefulness), course syllabi, } \\
\text { informal faculty discussion }\end{array}$ & $\begin{array}{l}\text { - In depth exploration } \\
\text { - Hands-on practice } \\
\text { - Project-based approach } \\
\text { - Modeling and course syllabi } \\
\text { design } \\
\text { - Concrete, content-area } \\
\text { examples } \\
\text { - Ongoing assessment of } \\
\text { progress } \\
\text { - Timesavers provided } \\
\text { - Workshop: addresses } \\
\text { individual needs; differentiation } \\
\text { - One-on-one assistance and } \\
\text { professional sharing } \\
\text { - Video production }\end{array}$ & $\begin{array}{l}\text { Evidence of effective techno } \\
\text { integration (change in course syllabi) } \\
\text { at an adequate level of technology }\end{array}$ \\
\hline
\end{tabular}


TABLE 1 | Continued

\begin{tabular}{|c|c|c|c|c|c|c|c|}
\hline References & $\begin{array}{l}\text { Research design } \\
\text { (methods) }\end{array}$ & $\begin{array}{l}\text { Sample } \\
(n)\end{array}$ & $\begin{array}{l}\text { Educational } \\
\text { context }\end{array}$ & $\begin{array}{l}\text { Aim(s) } \\
\text { of research }\end{array}$ & $\begin{array}{l}\text { Data sources } \\
\text { (investigated variables) }\end{array}$ & $\begin{array}{l}\text { Main characteristics of ETPD } \\
\text { project/program }\end{array}$ & $\begin{array}{l}\text { Identified } \\
\text { ETPD markers }\end{array}$ \\
\hline $\begin{array}{l}\text { Triggs and John } \\
\text { (2004) } \\
\text { (United Kingdom) }\end{array}$ & $\begin{array}{l}\text { Research and } \\
\text { Development } \\
\text { (qualitative) }\end{array}$ & 17 & $\begin{array}{l}\text { College of } \\
\text { Education, } \\
\text { primary and } \\
\text { secondary } \\
\text { schools } \\
\text { (Mathematics } \\
\text { and English } \\
\text { Departments) }\end{array}$ & $\begin{array}{l}\text { - Focus on development and } \\
\text { dissemination of professional } \\
\text { knowledge } \\
\text { - To re-model relationship } \\
\text { between practice and research }\end{array}$ & $\begin{array}{l}\text { Literature review, notes of } \\
\text { meetings, reports of the work of } \\
\text { the teams, video, interviews }\end{array}$ & $\begin{array}{l}\text { - Teams of teachers, TEs, and } \\
\text { researchers } \\
\text { - Opportunities to play and } \\
\text { experiment with ICT } \\
\text { - Fusing of members in subject } \\
\text { disciplines } \\
\text { - Recognition and acceptance } \\
\text { of plurality: complementary } \\
\text { roles ofteachers, TEs, and } \\
\text { researchers } \\
\text { - Design, enact, research } \\
\text { - Reflection based on video: key } \\
\text { link between } 3 \text { communities } \\
\text { - Communities of practice: } \\
\text { inter-relations in meso, micro, } \\
\text { and macro communities: } \\
\text { creation and dissemination of } \\
\text { professional knowledge }\end{array}$ & $\begin{array}{l}\text { - Role shifts, identity: from } \\
\text { constrained deliverers to enabled } \\
\text { professionals } \\
\text { - Conflicts, tensions } \\
\text { - Motivation, sense of engagement, } \\
\text { eager to take risks, sense of control, } \\
\text { ownership } \\
\text { - Knowledge as constructed, } \\
\text { situated, social, distributed: from } \\
\text { transaction to transformation } \\
\text { - Crucial nexus between knowledge } \\
\text { exchange and changes in practice } \\
\text { - Innovation: problem solving }\end{array}$ \\
\hline $\begin{array}{l}\text { Ulrich and } \\
\text { Karvonen (2011) } \\
\text { (USA) }\end{array}$ & $\begin{array}{l}\text { Structural } \\
\text { Equation Modeling } \\
\text { study (quantitative) }\end{array}$ & 285 & $\begin{array}{l}\text { Community } \\
\text { Colleges }\end{array}$ & $\begin{array}{l}\text { To test predictors of integration } \\
\text { of web } 2.0 \text { into formal online } \\
\text { learning environments }\end{array}$ & $\begin{array}{l}\text { Surveys (attitudes toward learner } \\
\text { self-direction, techno } \\
\text { acceptance and innovation, } \\
\text { interest, intended use, } \\
\text { knowledge, contextual } \\
\text { conditions) }\end{array}$ & $\begin{array}{l}\text { No combined ETPD project } \\
\text { Workshops: PD activities should } \\
\text { convey principles of learner } \\
\text { self-direction, facilitative } \\
\text { techniques, instructional } \\
\text { practices, with a mentoring } \\
\text { program (to support cycle of } \\
\text { ongoing evaluation, revision, } \\
\text { assistance) and a platform for } \\
\text { asynchronous and synchronous } \\
\text { PD activities }\end{array}$ & $\begin{array}{l}\text { - Intended use } \\
\text { - Interest } \\
\text { - Use of technology } \\
\text { - Control/ role: instructors giving } \\
\text { up control of the assessment } \\
\text { component (student control over } \\
\text { assessment of learning outcomes) } \\
\text { - Higher Personal Innovativeness in } \\
\text { the domain of Information } \\
\text { Technology (Agarwal and Prasad, } \\
\text { 1998): fewer positive perceptions } \\
\text { (toward technology use) required } \\
\text { than an individual who is } \\
\text { less innovative }\end{array}$ \\
\hline
\end{tabular}


learning, based on three principles: (1) encouraging multimodal instructional techniques and delivery methods, (2) providing learners with multiple means of action and expression, and (3) providing learners with multiple means of engagement in learning.

Researchers then noted two major consequences when adopting this faculty-as-learner posture. First, adopting this posture helped faculty members to move from a hierarchical relationship with their students or colleagues to a partnership model (Ashton and Newman, 2006; Archambault et al., 2010). For instance, in Seels et al. (2003) study, each member of the collaborative community of learners was placed in the position of both learning with and from other members of the community. Matthew et al. (2002) also observed that teacher educators, when paired with a student-coach in a mentoring process toward ETDP, experienced reversed roles and, consequently, the relationship between them changed along the mentoring process. Similarly, Baran (2016) revealed the importance of reciprocal learning between faculty members (positioned as mentees) and their graduate students (positioned as mentors). Archambault et al. (2010) also found that some faculty members who were placed in a learner role felt more like a "partner in learning" (p. 10); they consequently saw their students as contributors to knowledge and allowed their students to participate in the creation of knowledge content. For instance, because some faculty members felt themselves to be limited in technology knowledge, they accepted help from their most "techy" students. Some faculty members even completed the same project they had assigned to their students, and could admit that some students' projects were better than their own.

The second major consequence of this posture is that helping faculty members to gain a better understanding and awareness of learners' needs and profiles encourages self-management and self-efficacy (Ashton and Newman, 2006; Shattuck and Anderson, 2013) and brings faculty members closer to the learnercentered education inspired by Dewey (1933). In other words, experiencing the reverse side of the teaching and learning process (i.e., the learner side) leads faculty members to change their teaching posture (Shattuck and Anderson, 2013; Psiropoulos et al., 2016). Psiropoulos et al. (2016) suggested that "the ability to see oneself through the eyes of the learner" (p. 211) might be one of the most useful ways to positively impact ETPD. Shattuck and Anderson (2013) revealed that the learner posture helped faculty members to rethink their teaching practice, because "experiencing what it feels like to learn something unfamiliar and difficult is the best way to help teachers empathize with the emotions and feelings of their own learners as they begin to traverse new intellectual terrains" (pp. 199-200).

In conclusion, within this learner posture, some key conditions were recommended in the research literature for ETPD effectiveness: address learners' needs (Psiropoulos et al., 2016) and engage faculty in problem-oriented projects leading to practical solutions (Esterhuizen et al., 2013) and into authentic experiences, bearing in mind that quantity and quality of experiences influence ICT adoption (Esterhuizen et al., 2013).

\section{Faculty-As-Designer}

In their literature review, Becuwe et al. (2017) identified four main characteristics for an efficient design task in ETPD programs: innovativeness, concreteness, complexity, and realworld use. The design task is also usually understood as part of a developmental process, with the task used as an entry stage in action research (Chen et al., 2018) to trigger faculty's professional development in ETPD programs and/or to ultimately generate design principles in design-based research (Shattuck and Anderson, 2013).

Researchers provided two main reasons for adopting a faculty-as-designer posture (Becuwe et al., 2017; Mourlam, 2017; Avidov-Ungar and Forkosh-Baruch, 2018). First, learning technology by design (Koehler and Mishra, 2005a,b; Mishra and Koehler, 2006; Koehler et al., 2011) has been an early TPACK development approach in higher education (Mourlam, 2017), and is increasingly used as a strategy to familiarize teachers with the various components of the TPACK model (Becuwe et al., 2017). As a matter of fact, in their Delphi study, Becuwe et al. (2017) elicited the consensual idea that the design task in ETPD should have a long-term perspective and lead to a good understanding of both TPACK components and their interactions within the model. A second reason for adopting this posture is that designing class activities is one of the main tasks of faculty and therefore design is an essential component of their professional identity (Avidov-Ungar and Forkosh-Baruch, 2018).

In our review, we observed that ETPD training programs generated several categories of designed products. First, when positioned as designers, faculty members were given the opportunity through ETPD programs to create and develop their own resources within their teaching context, such as the redesign of a single lesson or course unit, in order to include social networking (Archambault et al., 2010) or learnercentered principles (Derting et al., 2016). Another example is the increased number of web-based learning environments, courses on Moodle, and ICT pedagogical initiatives produced by faculty members by the end of their ETPD program (Baya'a and Daher, 2015). Physical space and pedagogy have also been redesigned, together with efforts in technology training (Friel et al., 2009). Second, the design process also affected the tools used by faculty members. For instance, Chen et al. (2018) focused on the continuously designed and reviewed tools of a novice mathematics teacher educator-researcher. Similarly, Hoekstra and Crocker (2015) paid attention to the design of feedback tools for their collaboratively designed eportfolio approach. Third, some ETPD training programs have been co-designed (i.e., collaboratively designed with faculty members), focusing on incorporating principles of good practice in undergraduate education (Friel et al., 2009) or on technology integration (Teclehaimanot and Lamb, 2005). Involving faculty members in the design of their own training, Teclehaimanot and Lamb (2005) study goal was to reach the "ripple effect" of faculty redesigning their syllabi, revealing interconnections between the designed intervention and generated production. This leads us to the final category of designed products, 
research itself, which in recent times has increasingly been codesigned. Shattuck and Anderson (2013) observed the increased interest among educational researchers over the last decade in design-based research, defined as "a systematic but flexible methodology aimed to improve educational practices through iterative analysis, design, development, and implementation, based on collaboration among researchers and practitioners in real-world settings, and leading to contextually-sensitive design principles and theories" (Wang and Hannafin, 2005, pp. 6-7).

Whatever is being designed, most researchers have pointed out the importance of the collaborative dimension of the design process: design has mostly been used within ETPD as a teambased activity (e.g., Dolk et al., 2002; Shattuck and Anderson, 2013; Baya'a and Daher, 2015; Hoekstra and Crocker, 2015; Derting et al., 2016; Becuwe et al., 2017). According to Foley and Masingila (2014), this is because "without such collaboration, interventions are unlikely to affect changes in the real-world context" (p. 800). Even when projects were individually designed to better meet participants' needs and interests, researchers demonstrated how the creation of collaborative communities of learners was concomitant (Seels et al., 2003), or how the design process of a teacher educator was embedded and influenced by different collectives (Psycharis and Kalogeria, 2017). Becuwe et al. (2017) argued that "collaborative design (in teacher design teams) of technology-enhanced lessons has been shown to contribute to the development of competencies necessary to integrate technology in education" (p. 159). This is why the engagement of faculty members in design-based activities simultaneously enabled investigation of practice and fostered the creation of communities above the traditional gap between practitioners and researchers (Triggs and John, 2004; Foley and Masingila, 2014).

Finally, Mourlam (2017) observed that design-based research usually ended prior to the implementation of instruction, while Archambault et al. (2010) proposed engaging faculty in the full instructional design process, including the implementation phase. Recently, Jaipal-Jamani et al. (2018) extended the process of learning, designing, and implementing until the mentoring phase in which teacher educators adopt a technology leader's role in ETPD workshops.

\section{Faculty-As-Researcher}

Positioning faculty members in a researcher posture is grounded in action research methodology (Maor, 2006; García and Roblin, 2008; Archambault et al., 2010; Chen et al., 2018). Shattuck and Anderson (2013) noted that action research typically positions the teacher as a researcher: through a form of disciplined inquiry, a personal attempt is made to understand, improve, and reform practice. In action research, "the emphasis is on reflective research to inform individual practice at the local level" (Shattuck and Anderson, 2013, p. 189).

The centrality of faculty's reflexive posture, a feature common to both action research and design-based research methodology, can also be found more generally in most ETPD programs (Dolk et al., 2002; Shattuck and Anderson, 2013; Foley and Masingila, 2014; Hoekstra and Crocker, 2015; Mourlam, 2017; Jaipal-Jamani et al., 2018). Researchers widely refer to Schön $(1983,1987$, 1992) "reflective practitioner" approach as a central aspect of
ETPD programs in higher education. This reflective posture can be understood as (1) the ability to have critical discussions with peers, observing, sharing, and discussing experiences and practices (Dolk et al., 2002; Maor, 2006; Friel et al., 2009), or (2) a behavior toward self-oriented reflection (Drent and Meelissen, 2008; García and Roblin, 2008; Chen et al., 2018). García and Roblin (2008) indicated the strong research trend of self-study, explaining that teacher educators are simultaneously both "subject" and "object" of their own research.

Diverse techniques of data collection are used with the goal of activating and facilitating this self-reflection. According to Drent and Meelissen (2008), this stimulation of self-reflection appears to be one of the supportive conditions for personal entrepreneurship, and the ability to reflect on one's own behavior is an important teacher characteristic for the implementation of educational innovations. Chen et al. (2018) also suggested that the basis for facilitating professional learning and development is the teacher educator-researcher's continuous reflection upon his own practice.

Positioning faculty members as researchers also contributes to developing certain abilities while investigating professional practice: (1) the questioning capacity, (2) observation and communication skills, (3) awareness raising, (4) the ability to model, and (5) improving the quality of meaning and knowledge construction. Concerning the questioning capacity, questions were used as a starting point to design and develop a plan of action and intervention (Drent and Meelissen, 2008; García and Roblin, 2008), but "as a consequence of the resultant reflection processes, new questions emerged" (García and Roblin, 2008, p. 105). Observation was the first step in Dolk et al. (2002) ETPD approach. They demonstrated the necessity of developing communication skills, such as narrative knowledge, to enable participants to share and discuss observations that made them fully aware of the inaccuracy and incompleteness of their observations, which encouraged them to broaden and deepen their analysis of the observed situation. Psycharis and Kalogeria (2017) revealed "how observation and the subsequent reflective session facilitated trainees' transition from personal to professional instrumental genesis" (p. 8). García and Roblin (2008) used participant observation by a "critic friend" (a Ph-D student) as a "mirror" to encourage self-reflection. Adopting a researcher posture also raised faculty consciousness, which enabled changes to occur. Awareness is always sought through reflection, with the ultimate goal of transforming practice. Faculty awareness was purposely increased through this researcher posture, either for a better acknowledgment of their students' needs, interests, and motivations (Drent and Meelissen, 2008) or for a better acknowledgment of their own areas of improvement (Hoekstra and Crocker, 2015), because exercising self-awareness, combined with self-management and meta-learning (Kukulska-Hulme, 2012), may influence faculty's immediate (unconscious) behavior (Dolk et al., 2002). According to Dolk et al. (2002), this faculty awareness can help faculty members generalize the constructed situated practical knowledge into a didactic for teacher education, expanding their personal repertoire and allowing the construction of theory. This is how the researcher posture may develop the faculty ability of 
modeling (Christ et al., 2017), such as "role modeling" (KukulskaHulme, 2012; Rowe et al., 2013). Finally, a researcher posture may improve quality of meaning and knowledge construction (Chen et al., 2018). Dolk et al. (2002) demonstrated that reflection is a precondition to knowledge construction while others consider that strengthened ties between practice and theoretical construction of knowledge are crucial (Triggs and John, 2004; Drent and Meelissen, 2008; Kukulska-Hulme, 2012). More generally, the purpose of a researcher posture is to achieve in-depth comprehension, characterize processes and variables, and form explanations, linking participants' reflections on their experiences with "the experience per se" (e.g., Avidov-Ungar and Forkosh-Baruch, 2018, p. 186), with the ultimate purpose of promoting changes and faculty empowerment (García and Roblin, 2008).

Kukulska-Hulme (2012) concluded that "ultimately faculty need to plan and revisit their own development in a self-directed way within a community of learning (peers and/or students), taking into account the various aspects of their role and their own professional learning needs" (p. 253). She argued that faculty members should simultaneously adopt these three postures, reflecting the assumption that faculty members should develop their lifelong professional learning (the learner posture), teaching (the designer posture), and research (the researcher posture).

\section{CONCLUSION}

Conducting this literature review has given us the opportunity to explore ETPD in higher education through a diversity of methodologies and theories, navigating through several educational contexts in different areas of the world since 2002 . Following our immersion in this emerging field of research, we conclude by attempting to illuminate some key insights and suggest some prospects for future inquiry.

A first glance at our literature review demonstrates that AngloSaxon countries prevail (see Table 1), which may be explained by the bias toward English language publications. However, the Netherlands has stood out since the beginning of this emerging research field. In fact, in common with Anglo-Saxon countries, the Netherlands has implemented early national policies, which have brought up faculty ETPD in research agendas earlier than elsewhere. As noted by Drent and Meelissen (2008), "since the mid-1990's, the Dutch government has provided teacher education institutes with special facilities to play a pioneering role in the integration of ICT in education" (p. 187). The same facilitating national educational context can be found in the USA where the Department of Education initiated the Preparing Tomorrow's Teachers to Use Technology program in 1999. In Australia, Romeo et al. (2012) indicated that the Australian National Teaching Teachers for the Future program has engendered research opportunities based on its purpose to build the ICT education capacity of the next generation of teachers. These "pioneer" countries first investigated the field at the individual scale (Dolk et al., 2002), then at the one-toone (Matthew et al., 2002) and community scales (Maor, 2006). They were followed by "newcomer" countries including Spain
(García and Roblin, 2008), Canada (Shattuck and Anderson, 2013), South Africa (Esterhuizen et al., 2013), Kenya (Foley and Masingila, 2014), Turkey (Baran, 2016), Israël (Baya’a and Daher, 2015), United Arab Emirates (Psiropoulos et al., 2016), Belgium (Becuwe et al., 2017), Greece (Psycharis and Kalogeria, 2017), and Taïwan (Chen et al., 2018). These countries offered the opportunity to extend our knowledge in this field of research to different geographical and cultural areas and, therefore, to different educational contexts. From a theoretical perspective, the first works in the field were based on technology, and a major trend toward innovation-based theoretical frameworks then took the lead in all pioneer countries. Finally, beginning in 2013, we noticed an increasing number of international collaborations to investigate faculty ETPD. For example, authors from three different countries (USA, Netherlands, and Belgium) collaborated in Becuwe et al. (2017) study. Conducting this literature review has allowed us to follow the emergence and worldwide spread of an emerging field of research demonstrating that a wide range of theoretical and methodological approaches have been adopted.

Whatever theoretical approaches researchers adopted in this field of knowledge (i.e., technology-based, innovation-based, or socio-cultural interactions-based), they share the same vision of a sustainable and scalable educational change, enabling faculty members to achieve the new educational requirements of the twenty-first century. Niederhauser et al. (2018) defined sustainability and scalability, respectively, as "a persistent and ongoing change of the educational culture" (p. 509) and as "the likelihood that an innovation will diffuse effectively across a culture/context" (p. 511). This review may help inform stakeholders and policy makers to promote a sustainable and scalable educational change by highlighting key markers of faculty ETPD from empirical evidence. The need to reframe faculty members' pedagogies toward a more student-centered constructivist approach is one of the key markers (e.g., Ashton and Newman, 2006; Rienties et al., 2013), echoing what we know about the impact on teachers' pedagogy of technology integration in the classroom (Fishman and Dede, 2016). According to these authors, educational technology should encourage us to rethink the teaching and learning process as a whole and lead beyond what is achievable without it. Therefore, a strong and authentic feeling of transformation in faculty's own practice is considered a valuable criterion of success for ETPD programs (García and Roblin, 2008). Some authors have also highlighted changed attitudes toward ICT (King and Boyatt, 2014; Baya'a and Daher, 2015), a shift of beliefs and perceptions (Psiropoulos et al., 2016), changed roles in the classroom (Esterhuizen et al., 2013), or improved knowledge and competencies in technology-enhanced teaching (Jaipal-Jamani et al., 2018). Another key marker of faculty ETPD is the redesign of the course curriculum (e.g., Matthew et al., 2002). As faculty members become involved in ETPD programs, authors have observed that (1) new resources have been created such as portfolios or videos (Ashton and Newman, 2006; Christ et al., 2017), (2) the number of webbased learning environments, courses on Moodle, and ICT pedagogical initiatives has increased (Baya'a and Daher, 2015), or (3) a wider variety of ways to integrate technology in 
teaching have been used (Matthew et al., 2002). Finally, perceived impacts on students emerged sparingly as a key marker in our literature review (Archambault et al., 2010; Derting et al., 2016). This is surprising because students' success should be the ultimate goal of faculty members' ETPD. Therefore, perceived impacts on students should be used as a basic outcome to improve faculty professional development. Derting et al. (2016) raised the lack of objective measures of student learning and skills.

As we conclude and reflect on this journey in an emerging field of research, we realize that while, on the one hand, we have gained much knowledge about ETPD in higher education, much uncertainty remains. For example, we do not know (1) if faculty members' postures should be adopted simultaneously or through a developmental process, (2) if these different postures independently affect key markers of ETPD or target specific ones, or (3) if future research in this field should reach a consensus regarding which theoretical and methodological approach should be adopted in order to build an inclusive framework to study ETPD in higher education within a variety of diverse educational contexts. We suggest design-based implementation research (Fishman and Dede, 2016) to be this inclusive framework as it will help (1) understand faculty members ETPD beyond the three separate main postures we identified, (2) consider sustainability and scalability as key issues from the beginning of the design process, and (3) focus at the level of schools or school systems

\section{REFERENCES}

Agarwal, R., and Prasad, J. (1998). A conceptual and operational definition of personal innovativeness in the domain of information technology. Information Syst. Res. 9, 204-215. doi: 10.1287/isre.9.2.204

Agyei, D. D., and Voogt, J. M. (2011). Exploring the potential of the will, skill, tool model in Ghana: predicting prospective and practicing teachers' use of technology. Comput. Educ. 56, 91-100. doi: 10.1016/j.compedu.2010.08.017

Archambault, L., Wetzel, K., Foulger, T. S., and Kim Williams, M. (2010). Professional development 2.0: transforming teacher education pedagogy with 21 st century tools. J. Digital Learn. Teach. Educ. 27, 4-11. doi: $10.1080 / 21532974.2010 .10784651$

Ashton, J., and Newman, L. (2006). An unfinished symphony: $21^{\text {st }}$ century teacher education using knowledge creating heutagogies. Br. J. Educ. Technol. 37, 825-840. doi: 10.1111/j.1467-8535.2006.00662.x

Avidov-Ungar, O., and Forkosh-Baruch, A. (2018). Professional identity of teacher educators in the digital era in light of demands of pedagogical innovation. Teach. Teach. Educ. 73, 183-191. doi: 10.1016/j.tate.2018.03.017

Bai, H., and Lehman, J. (2003). "Impact of a professional development project on university faculty members' perceptions and use of technology," in Proceedings of ED-MEDIA-World Conference on Educational Multimedia, Hypermedia \& Telecommunications, 2003, eds D. Lassner and C. McNaught. (Honolulu, HI: Association for the Advancement of Computing in Education), 1927-1934. Available online at: https://www.learntechlib.org/p/14126 (accessed January 6, 2020).

Baran, E. (2016). Investigating faculty technology mentoring as a universitywide professional development model. J. Comput. Higher Educ. 28, 45-71. doi: 10.1007/s12528-015-9104-7

Baya'a, N., and Daher, W. (2015). The development of college instructors' technological pedagogical and content knowledge. Procedia Soc. Behav. Sci. 174, 1166-1175. doi: 10.1016/j.sbspro.2015.01.733

Becuwe, H., Pareja Roblin, N., Tondeur, J., Thys, J., Castelein, E., and Voogt, J. (2017). Conditions for the successful implementation of teacher educator as opposed to at the level of a single classroom or group of classrooms.

Finally, conducting a systematic literature review in ETPD in higher education brings out some limitations related to an emerging field of research including potential number of papers that may be included, diversity of research investigations, and dealing with a rapidly changing technology world that impacts research inquiries. However, this attempt to synthetize in a meaningful way research in ETPD in higher education may offer to the research community the opportunity to build their investigations on what we already know in this emerging field of research in order to explore what remains to be discovered.

\section{AUTHOR CONTRIBUTIONS}

SL conducted the systematic literature review, data collection and data analysis, prepared summary documents, and drafted the manuscript. DP participated to data collection, data analysis, and proofread the manuscript. All authors have read and approved the final version of the manuscript, and agree with the order of presentation of the authors.

\section{FUNDING}

This research did not receive any specific grant from funding agencies in the public, commercial, or not-for-profit sectors.

design teams for ICT integration: a Delphi study. Austral. J. Educ. Technol. 33, 159-172. doi: 10.14742/ajet.2789

Bruner, J. S. (1975). The ontogenesis of speech acts. J. Child Lang. 2, 1-19. doi: 10.1017/S0305000900000866

Chen, J.-C., Lin, F.-L., and Yang, K.-L. (2018). A novice mathematics teacher educator-researcher's evolution of tools designed for in-service mathematics teachers' professional development. J. Math. Teach. Educ. 21, 517-539. doi: 10.1007/s10857-017-9396-9

Christ, T., Arya, P., and Chiu, M. M. (2017). Video use in teacher education: an international survey of practices. Teach. Teach. Educ. 63, 22-35. doi: $10.1016 /$ j.tate.2016.12.005

Coughlin, E., and Lemke, C. (1999). Professional Competency Continuum. Professional Skills for the Digital Age Classroom. Santa Monica, CA:: Milken Exchange on Education Technology. Available online at: https://www. mff.org/assets/Uploads/newsroom_archive/publications/ME159.pdf (accessed September 11, 2019).

Davis, F. D. (1989). Perceived usefulness, perceived ease of use, and user acceptance of information technology. MIS Q. 13, 319-340. doi: 10.2307/ 249008

Dawson, V. (2008). Use of information and communication technology by early career science teachers in Western Australia. Int. J. Sci. Educ. 30, 203-219. doi: 10.1080/09500690601175551

Derting, T. L., Ebert-May, D., Henkel, T. P., Maher, J. M., Arnold, B., and Passmore, H. A. (2016). Assessing faculty professional development in STEM higher education: Sustainability of outcomes. Sci. Adv. 2:e1501422. doi: $10.1126 /$ sciadv.1501422

Dewey, J. (1933). "Why reflective thinking must be an educational aim," in How We Think: A Restatement of the Relation of Reflective Thinking to the Educative Process (New York, NY: D.C. Heath and Co), 17-34.

Dolk, M., den Hertog, J., and Gravemeijer, K. (2002). Using multimedia cases for educating the primary school mathematics teacher educator: a design study. Int. J. Educ. Res. 37, 161-178. doi: 10.1016/S0883-0355(02) 00058-7 
Drent, M., and Meelissen, M. (2008). Which factors obstruct or stimulate teacher educators to use ICT innovatively? Comput. Educ. 51, 187-199. doi: 10.1016/j.compedu.2007.05.001

Education Services Australia (2012). Teaching Teachers for the Future: Building the ICTE Capacity of Pre-Service Teachers in Australian Institutions. Sidney, BC. Available online at: https://www.voced.edu.au/content/ngv\%3A54501 (accessed September 11, 2019).

Esterhuizen, H. D., Blignaut, S., and Ellis, S. (2013). Looking out and looking in: Exploring a case of faculty perceptions during e-learning staff development. Int. Rev. Res. Open Distrib. Learn. 14, 59-80. doi: 10.19173/irrodl.v14i3.1358

Fishman, B., and Dede, C. (2016). "Teaching and technology: new tools for new times," in Handbook of Research on Teaching, $5^{\text {th }}$ Edn., eds D. H. Gitomer and C. A. Bell (Washington, DC. American Educational Research Association), 1269-1334.

Foley, A. R., and Masingila, J. O. (2014). Building capacity: challenges and opportunities in large class pedagogy (LCP) in Sub-Saharan Africa. Higher Educ, 67, 797-808. doi: 10.1007/s10734-013-9697-6

Friel, T., Britten, J., Compton, B., Peak, A., Schoch, K., and van Tyle, W. K. (2009). Using pedagogical dialogue as a vehicle to encourage faculty technology use. Comput. Educ. 53, 300-307. doi: 10.1016/j.compedu.2009.02.002

Gamire, E., Pearson, G., and Committee on Assessing Technological Literacy in the United States and National Academy of Engineering and National Research Council. (2006). Tech tally: Approaches to Assessing Technological Literacy. Washington, DC: The National Academies Press. Available online at: https:// books.google.fr/books?id=L90vRE-bCY4C (accessed September 11, 2019).

García, L. M., and Roblin, N. P. (2008). Innovation, research and professional development in higher education: learning from our own experience. Teach. Teach. Educ. 24, 104-116. doi: 10.1016/j.tate.2007.03.007

Gronseth, S., Brush, T., Ottenbreit-Leftwich, A., Strycker, J., Abaci, S., Easterling, W., et al. (2010). Equipping the next generation of teachers: technology preparation and practice. J. Digital Learn. Teach. Educ. 27, 30-36. doi: 10.1080/21532974.2010.10784654

Hagner, P. R., and Schneebeck, C. (2001). "Engaging the faculty," in Educause Leadership Strategies. Technology-Enhanced Teaching and Learning: Leading and Supporting the Transformation on Your Campus, Vol. 5, eds C. A. Barone and P. R. Hagner (San Francisco, CA: Jossey-Bass Publishing), 1-12.

Harris, J., Mishra, P., and Koehler, M. (2009). Teachers' technological pedagogical content knowledge and learning activity types: curriculumbased technology integration reframed. J. Res. Technol. Educ. 41, 393-416. doi: 10.1080/15391523.2009.10782536

Hoekstra, A., and Crocker, J. R. (2015). Design, implementation, and evaluation of an ePortfolio approach to support faculty development in vocational education. Stud. Educ. Eval. 46, 61-73. doi: 10.1016/j.stueduc.2015.03.007

Jaipal-Jamani, K., Figg, C., Collier, D., Gallagher, T., Winters, K.-L., and Ciampa, K. (2018). Developing TPACK of university faculty through technology leadership roles. Ital. J. Educ. Technol. 26, 39-55. doi: 10.17471/2499-4324/984

Jorgensen, M., Havel, A., Fichten, C., King, L., Marcil, E., Lussier, A., et al. (2018). "Simply the best": professors nominated by students for their exemplary technology practices in teaching. Educ. Information Technol. 23, 193-210. doi: 10.1007/s10639-017-9594-1

Keengwe, J., Kidd, T., and Kyei-Blankson, L. (2009). Faculty and technology: Implications for faculty training and technology leadership. J. Sci. Educ. Technol. 18, 23-28. doi: 10.1007/s10956-008-9126-2

King, E., and Boyatt, R. (2014). Exploring factors that influence adoption of elearning within higher education: factors that influence adoption of e-learning. Br. J. Educ. Technol. 46, 1272-1280. doi: 10.1111/bjet.12195

King, K. P. (2002). Educational technology professional development as transformative learning opportunities. Comput. Educ. 39, 283-297. doi: 10.1016/S0360-1315(02)00073-8

Koehler, M. J., and Mishra, P. (2005a). Teachers learning technology by design. J. Comput. Teach. Educ. 21, 94-102. doi: 10.1080/10402454.2005.10784518

Koehler, M. J., and Mishra, P. (2005b). What happens when teachers design educational technology? The development of technological pedagogical content knowledge. J. Educ. Comput. Res. 32, 131-152. doi: 10.2190/0EW7-01WB-BKHL-QDYV

Koehler, M. J., Mishra, P., Bouck, E. C., DeSchryver, M., Kereluik, K., Shin, T. S., et al. (2011). Deep-play: developing TPACK for 21st century teachers. Int. J. Learn. Technol. 6, 146-163. doi: 10.1504/IJLT.2011.042646
Koehler, M. J., Mishra, P., and Yahya, K. (2007). Tracing the development of teacher knowledge in a design seminar: Integrating content, pedagogy and technology. Comput. Educ. 49, 740-762. doi: 10.1016/j.compedu.2005.11.012

Kukulska-Hulme, A. (2012). How should the higher education workforce adapt to advancements in technology for teaching and learning? Internet Higher Educ. 15, 247-254. doi: 10.1016/j.iheduc.2011.12.002

Lameul, G. (2008). Les effets de l'usage des technologies d'information et de communication en formation d'enseignants, sur la construction des postures professionnelles. [The effects of ICT use in teacher education, for the construction of professional postures]. Savoirs 17, 71-94. doi: $10.3917 /$ savo.017.0071

Lave, J. (1988). Cognition in Practice: Mind, Mathematics, and Culture in Everyday Life. Cambridge: Cambridge University Press.

Lave, J., and Wenger, E. (1991). Situated Learning: Legitimate Peripheral Participation. Cambridge: Cambridge University Press.

Maor, D. (2006). Using reflective diagrams in professional development with university lecturers: a developmental tool in online teaching. Internet Higher Educ. 9, 133-145. doi: 10.1016/j.iheduc.2006.03.005

Matthew, K., Stephens, E., Callaway, R., Letendre, C., and Kimbell-Lopez, K. (2002). Adoption of information communication technology by teacher educators: One-on-one coaching. J. Information Technol. Teach. Educ. 11, 45-62. doi: 10.1080/14759390200200122

Mauss, M. (1990). The Gift: The Form and Reason for Exchange in Archaic Societies. New York, NY; London, UK: W.W. Norton and Company.

Mishra, P., and Koehler, M. J. (2006). Technological pedagogical content knowledge: a framework for teacher knowledge. Teach. Coll. Rec. 108, 1017-1054. doi: 10.1111/j.1467-9620.2006.00684.x

Mitra, S., Dangwal, R., Chatterjee, S., Jha, S., Bisht, R., and Kapur, P. (2005). Acquisition of computing literacy on shared public computers: children and the "hole in the wall." Austral. J. Educ. Technol. 21, 407-426. doi: 10.14742/ ajet. 1328

Mourlam, D. (2017). Preparing for infusion: emergence of a model for faculty TPACK development. J. Technol. Teach. Educ. 35, 301-325. Available online at: https://www.learntechlib.org/primary/p/177836/ (accessed January 6, 2020).

Niederhauser, D. S., Howard, S. K., Voogt, J., Agyei, D. D., Laferriere, T., Tondeur, J., et al. (2018). Sustainability and scalability in educational technology initiatives: research-informed practice. Technol. Knowl. Learn. 23, 507-523. doi: 10.1007/s10758-018-9382-Z

Phuong T., Catherine Cole, S., and Zarestky, J. (2018). A systematic literature review of faculty development for teacher educators. Higher Educ. Res. Dev. 37, 373-389. doi: 10.1080/07294360.2017.1351423

Piaget, J. (1972). To Understand Is to Invent: The Future of Education. New York, NY: Grossman Publishers.

Psiropoulos, D., Barr, S., Eriksson, C., Fletcher, S., Hargis, J., and Cavanaugh, C. (2016). Professional development for iPad integration in general education: staying ahead of the curve. Educ. Information Technol. 21, 209-228. doi: $10.1007 /$ s10639-014-9316-x

Psycharis, G., and Kalogeria, E. (2017). Studying the process of becoming a teacher educator in technology-enhanced mathematics. J. Math. Teach. Educ. 21, 631-660. doi: 10.1007/s10857-017-9371-5

Puentedura, R. (2010). SAMR and TPCK: Intro to Advanced Practice. Available online at: http://hippasus.com/resources/sweden2010/SAMR_TPCK IntroToAdvancedPractice.pdf (accessed September 11, 2019).

Reyes, V. C., Reading, C., Doyle, H., and Gregory, S. (2017). Integrating ICT into teacher education programs from a TPACK perspective: exploring perceptions of university lecturers. Comput. Educ. 115, 1-19. doi: 10.1016/j.compedu.2017.07.009

Rienties, B., Brouwer, N., and Lygo-Baker, S. (2013). The effects of online professional development on higher education teachers' beliefs and intentions towards learning facilitation and technology. Teach. Teach. Educ. 29, 122-131. doi: $10.1016 /$ j.tate.2012.09.002

Rogers, E. M. (1995). Diffusion of Innovations, 4th Edn.. New York, NY: Free Press. Romeo, G., Lloyd, M., and Downes, T. (2012). Teaching teachers for the future (TTF): building the ICT in education capacity of the next generation of teachers in Australia. Austral. J. Educ. Technol. 28, 949-964. doi: 10.14742/ajet.804

Rowe, M., Frantz, J., and Bozalek, V. (2013). Beyond knowledge and skills: the use of a Delphi study to develop a technology-mediated teaching strategy. BMC Med. Educ. 13, 1-8. doi: 10.1186/1472-6920-13-51 
Russell, C. (2009). A systemic framework for managing e-learning adoption in campus universities: individual strategies in context. ALT J Res. Learn. Technol. 17, 3-19. doi: 10.3402/rlt.v17i1.10771

Schön, D. A. (1983). The Reflective Practitioner: How Professionals Think in Action. New York, NY: Basic Books.

Schön, D. A. (1987). Educating the Reflective Practitioner. San Francisco, CA: Jossey-Bass.

Schön, D. A. (1992). Designing as reflective conversation with the materials of a design situation. Res. Eng. Design 3, 131-147. doi: 10.1007/BF01580516

Seels, B., Campbell, S., and Talsma, V. (2003). Supporting excellence in technology through communities of learners. Educ. Technol. Res. Dev. 51, 91-104. doi: $10.1007 / \mathrm{BF} 02504520$

Shattuck, J., and Anderson, T. (2013). Using a design-based research study to identify principles for training instructors to teach online. Int. Rev. Res. Open Distrib. Learn. 14, 186-210. doi: 10.19173/irrodl.v14i5.1626

Sher, W., Williams, A., and Northcote, M. (2015). The lived experience of online educators: insights from construction management. Construct. Econ. Build. 15, 49-62. doi: 10.5130/AJCEB.v15i2.4398

Shulman, L. S. (1986). Those who understand: knowledge growth in teaching. Educ. Res. 15, 4-14. doi: 10.3102/0013189X015002004

Shulman, L. S. (1987). Knowledge and teaching: foundations of the new reform. Harvard Educ. Rev. 57, 1-23. doi: 10.17763/haer.57.1.j463w79r56455411

Teclehaimanot, B., and Lamb, A. (2005). Technology-rich faculty development for teacher educators: the evolution of a program. Contemp. Issues Technol. Teach. Educ. 5, 330-344. Available online at: https://www.citejournal.org/ volume-5/issue-3-05/current-practice/technology-rich-facultydevelopmentfor-teacher-educators-the-evolution-of-a-program (accessed September 11, 2019).

Tondeur, J., van Braak, J., Ertmer, P. A., and Ottenbreit-Leftwich, A. (2017). Understanding the relationship between teachers' pedagogical beliefs and technology use in education: a systematic review of qualitative evidence. Educ. Technol. Res. Dev. 65, 555-575. doi: 10.1007/s11423-016-9481-2

Tondeur, J., van Braak, J., Sang, G., Voogt, J., Fisser, P., and OttenbreitLeftwich, A. (2012). Preparing pre-service teachers to integrate technology in education: a synthesis of qualitative evidence. Comput. Educ. 59, 134-144. doi: 10.1016/j.compedu.2011.10.009

Triggs, P., and John, P. (2004). From transaction to transformation: information and communication technology, professional development and the formation of communities of practice: from transaction to transformation. J. Comput. Assist. Learn. 20, 426-439. doi: 10.1111/j.1365-2729.2004.00101.x

Uerz, D., Volman, M., and Kral, M. (2018). Teacher educators' competences in fostering student teachers proficiency in teaching and learning with technology: an overview of relevant research literature. Teach. Teach. Educ. 70, 12-23. doi: 10.1016/j.tate.2017.11.005
Ulrich, J., and Karvonen, M. (2011). Faculty instructional attitudes, interest, and intention: predictors of Web 2.0 use in online courses. Internet Higher Educ. 14, 207-216. doi: 10.1016/j.iheduc.2011.07.001

UNESCO (United Nations Educational Scientific and Cultural Organization) (2002). Information and Communication Technology in Education: A Curriculum for Schools and Programme of Teacher Development. Paris. Available online at: https://unesdoc.unesco.org/ark:/48223/pf0000129538 (accessed September 11, 2019).

US Department of Education (1999). Preparing Tomorrow's Teachers to Use Technology program (PT3). Washington, DC. Available online at: https://www2. ed.gov/programs/teachtech/index.html (accessed September 11, 2019).

Venkatesh, V., and Brown, S. A. (2001). A longitudinal investigation of personal computers in homes: adoption determinants and emerging challenges. MIS $Q$. 25, 71-102. doi: 10.2307/3250959

Vygotsky, L. S. (1978). Mind in Society: The Development of Higher Psychological Processes. Cambridge, MA: Harvard University Press.

Wang, F., and Hannafin, M. J. (2005). Design-based research and technology-enhanced learning environments. Educ. Technol. Res. Dev. 53, 5-23. doi: 10.1007/BF025 04682

Wenger, E. (1998). Communities of Practice: Learning, Meaning and Identity. Cambridge, UK: Cambridge University Press.

Wenger, E., McDermott, R., and Snyder, W. M. (2002). Cultivating Communities of Practice: A Guide to Managing Knowledge. Boston, MA: Harvard Business School Press.

Wenger-Trayner, E. and Wenger-Trayner, B. (2015). Introduction to Communities of Practice. A Brief Overview of the Concept and Its Uses. Available online at: https://wenger-trayner.com/introduction-to-communities-of-practice/ (accessed September 11, 2019).

Wentworth, N., Graham, C. R., and Monroe, E. E. (2009). “TPACK development in a teacher education program," in Handbook of Research on New Media Literacy at the K-12 Level, eds L. T. W. Hin and R. Subramaniam (Hershey, PA: Information Science Reference), 823-838.

Conflict of Interest: The authors declare that the research was conducted in the absence of any commercial or financial relationships that could be construed as a potential conflict of interest.

Copyright () 2020 Lidolf and Pasco. This is an open-access article distributed under the terms of the Creative Commons Attribution License (CC BY). The use, distribution or reproduction in other forums is permitted, provided the original author(s) and the copyright owner(s) are credited and that the original publication in this journal is cited, in accordance with accepted academic practice. No use, distribution or reproduction is permitted which does not comply with these terms. 